\title{
Exploring the preinstruction and postinstruction non-Newtonian world views as measured by the Force Concept Inventory
}

\author{
Philip Eaton, Kinsey Vavruska, and Shannon Willoughby \\ Department of Physics, Montana State University, 1325-1399 S 6th Ave, Bozeman, Montana 59715, USA
}

(Received 20 December 2018; published 25 April 2019)

\begin{abstract}
Implementing targeted instructional techniques requires an understanding of the initial alternative world views students possess. These alternative world views can be measured using validated conceptual inventories combined with factor analysis methodologies. Using exploratory factor analysis and network module analysis, Scott and Schumayer and Brewe, Bruun, and Bearden identified the most prevalent and "rigid" of these world views which remained in the postinstruction student responses to the Force Concept Inventory. This paper seeks to identify the alternative world views that exist for students before and after instruction through the use of factor analysis applied to pre- and postinstruction responses from 19727 matched, student responses. Using the distractor taxonomy proposed by Hestenes, Wells, and Swackhamer, the prevalent alternative world views of these students are characterized and analyzed. We find that the pre-instruction and postinstruction coherent alternative world views are similar, suggesting they are learned by the students prior to instruction. Using a modified version of item response curves, it was found that the active force misconception is the most rigid of the misconceptions found pre- and postinstruction, and shows minor growth in these response rates at the end of instruction. The results of this study are consistent with what experienced instructors observe when determining what physics preconceptions incoming students have. This study's agreement, combined with the results of Scott and Schumayer and Brewe, Bruun, and Bearden, strengthens the physics education research community's suggestions for improving physics instruction when using only anecdotal or qualitative evidence.
\end{abstract}

DOI: 10.1103/PhysRevPhysEducRes.15.010123

\section{INTRODUCTION}

When a conceptual assessment is written, the distractors are generally created to target specific sets of misconceptions based on interviews and open-ended written responses from a small sample of students. Thus, it is important to determine whether or not the distractors within an assessment encompass misconceptions held by other, larger student samples. The Force Concept Inventory (FCI) is one of the most commonly used multiple choice surveys for assessing the kinematics and dynamics conceptual understanding of students in introductory mechanics classes [1]. The FCI has had numerous statistical analysis methods applied to it over the years, see Refs. [2-11], however, many of these investigations sought to better understand the coherent nonexpert world views, otherwise known as alternative world views, held by the students

*philip.eaton@montana.edu

Published by the American Physical Society under the terms of the Creative Commons Attribution 4.0 International license. Further distribution of this work must maintain attribution to the author(s) and the published article's title, journal citation, and DOI. either pre- or post-test. We are aware of only the studies performed by Scott and Schumayer and Brewe, Bruun, and Bearden which looked explicitly at the alternative world views held by students postinstruction; see Refs. [10,11], respectively.

According to Scott and Schumayer [10] and Brewe, Bruun, and Bearden [11], impetus, active force, and active agent misconceptions are the most prevalent and strongest held misconceptions by students after instruction, as measured by the FCI. The impetus misconception is the idea that impetus is a property an object has that causes its motion and can be dissipated, built up over time, etc. An active force misconception is the idea that when an object is in motion there must be a cause for that motion in the form of a force. Last, the active agent misconception is the idea that when two objects are pushing against one another, the object that is doing the pushing is applying a greater force onto the other. Without understanding the original misconceptions, or alternative world views held by students before instruction, it is difficult to determine whether or not they were "learned" due to instruction. This study monitored the evolution of world views by analyzing matched pretest and post-test student responses using the methods 
established by Scott and Schumayer and a variation of item response curves [10,12].

The analysis done in 2017 by Scott and Schumayer evaluated postinstruction misconceptions. This was done by treating the FCI as a 150 item assessment, where every response option for each question was treated as an individual item. From there, they used exploratory factor analysis to find the correlational groupings of the response options and then subgrouped each of the factors found using hierarchical cluster analysis. From these subgroupings Scott and Schumayer were able to identify the coherent alternative world views held by the students after instruction. In a network analysis of these factors, Scott and Schumayer identified the "motion implies force/force implies motion" idea as the prominent misconception students retained postinstruction within their sample [13]. They recommend spending more time during instruction to address this issue directly to help students overcome the misconception.

To support the results found by Scott and Schumayer, consider the study done by Brewe, Bruun, and Bearden in 2016. In this study, Brewe, Bruun, and Bearden used network analysis and the InfoMap community detection algorithm to identify groupings of responses by students postinstruction [11]. They found that impetus and "more force yields more result" were the primary misconception groupings. Both of these studies produced similar results for the alternative conceptions held by students postinstruction using two different statistical techniques on two separate student samples.

With a better understanding of the pre- and postinstruction misconceptions held by students, instructors will be able to spend more time targeting these misconceptions in an effort to work with students to overcome them. It is understood that most instructors will already be aware of many of the findings from this study, especially to those with experience working with introductory mechanics students. However, the quantitative verification of anecdotal and qualitative knowledge is vital to moving physics education research forward. This study seeks to supply evidence for students' pre-instruction world views in a similar statistical manner as Scott and Schumayer [10] and to monitor how these world views change from preto postinstruction. This will be done by answering the following research questions: (i) What misconceptions are the most prevalent and coherent from a factor analysis perspective for pre- and postinstruction student responses? (ii) Which misconceptions remained unchanged, corrected, and/or were taught over the course of instruction?

This article is organized as follows: first an explanation of the data and methodology used in this study can be found in Secs. II and III. The results of the factor analysis and the IRDCs are presented in Sec. IV. Finally, limitations for this study and future works are discussed in Secs. V, VI, and the
TABLE I. Test statistics for the pre- and post-test FCI data.

\begin{tabular}{lccccc}
\hline \hline & $N$ & Average & S.D. & \multicolumn{1}{c}{ Skew } & Kurtosis \\
\hline Pretest & 19727 & 0.437 & 0.213 & 0.632 & -0.330 \\
Post-test & 19727 & 0.609 & 0.221 & -0.096 & -0.924 \\
\hline \hline
\end{tabular}

conclusion in Sec. VII details the results and implications of this study.

\section{DATA}

Matched pre-post FCI data was supplied to the authors from Physport upon request and Institutional Review Board approval [14]. The data used in this study is a mixture of calculus- and algebra-based introductory Physics I courses (i.e., first-time mechanics courses) from post-secondary education institutions. Data of this type automatically marginalizes over variables like mathematical sophistication of the classes, teaching methods used, etc. This means that any conclusions made using this sample will be applicable to a general introductory mechanics student population. This assumes that the misconception factor structure for the students' responses found in this study is independent of the mathematical sophistication of the course taken by the students.

From the data, any students with blank responses were removed from the sample and only the remaining students that had both a pretest and post-test response vector were retained. This treatment of the data left the sample with 19727 matched student responses for both pre- and posttest implementations of the FCI. The pre-post test statistics can be found in Table I.

Instead of grading each item dichotomously, we followed the method proposed by Scott and Schumayer in Ref. [10] and treated each response option as an item on the assessment. This treatment makes the FCI a 150 item assessment (30 questions $\times 5$ options). The grading of the data was done in a dichotomous manner, where "true" (coded as a 1) indicates the option was selected by the student, and "false" (coded as a 0 ) if the option was not selected. The response vectors of the students treated in this manner are the data that were used for the factor analysis performed in the study.

\section{METHODOLOGY}

The first two sections will briefly discuss the process used to regularize the correlation matrix used for factor analysis. The methods used in this paper were first presented by Scott and Schumayer [10], where a more detailed description of the methodology can be found.

\section{A. Regularization and treatment of the correlation matrix}

To calculate the correlation of the response options (which are binary, categorical, true or false vectors) a 
tetrachoric correlation will need to be used. In this case, a Pearson $r$ or Spearman $\rho$ correlation would not be appropriate because the categorizations do not represent a discretization of an underlying continuous variable, thus the need for the tetrachoric correlation to produce a proper correlation matrix. When the correlation matrix is formed in this manner, the options within the same questions will inherently be anticorrelated due to the limited response format of the assessment. A student who selects option 1.A will inadvertently not be able to select options 1.B-1.E, which will be reflected as a strong anticorrelation between these options. These artificial anticorrelations will be removed from the correlation matrix by setting the off diagonals to zero within each question's $5 \times 5$ block. This is equivalent to replacing the main diagonal of the correlation matrix with $5 \times 5$ identity matrices set corner to corner to one another. As was indicated by Scott and Schumayer, this method seems a little ad hoc, however, the conceptual motivation behind the elimination of these anticorrelations is logically consistent. As a result, for one item's correct response option to appear in the same correlational grouping as one of its own distractors requires a link through an option from a separate item. As such, the formed groupings must be robust and coherent, otherwise this second party linking would be unlikely to occur.

Because of the removal of the artificially negative correlations and utilization of a tetrachoric correlation, the resulting correlation matrix is not guaranteed to be positive definite, so it might have negative eigenvalues. Since the correlation matrix is an $n \times n$ square matrix, $n$ eigenvalues ( $\left.\left\{\lambda_{i}\right\}\right)$ with related eigenvectors $\left(\left\{\mathbf{q}_{i}\right\}\right)$ can be generated for the matrix. The eigenvalues for the correlation matrix will be a mixture of positive and negative values, thus making it nonpositive definite. Methods to correct for the nonpositive definite nature of these correlation matrices have been proposed in Refs. [15-17]. This study, identical to the approach used by Scott and Schumayer, uses an eigenvalue decomposition of the correlation matrix, and recreates a positive-semidefinite matrix (i.e., a matrix whose eigenvalues are all greater than or equal to zero) using only the positive eigenvalues and their associated eigenvectors:

$$
S=\sum_{i=1}^{n} \lambda_{i} \mathbf{q}_{i} \cdot \mathbf{q}_{i}^{T} \approx \sum_{0 \leq \lambda_{i}} \lambda_{i} \mathbf{q}_{i} \cdot \mathbf{q}_{i}^{T}=S^{*},
$$

where $S$ is the original, reduced correlation matrix (the within-item anticorrelations removed) and $S^{*}$ is the recreated positive, semidefinite correlation matrix. $S^{*}$ will be used in the factor analysis for the remainder of the study. We now turn our attention to how factor analysis was performed on this correlation matrix and how the number of factors to be extracted was identified.

\section{B. Response option exploratory factor analysis}

Factor analysis, specifically exploratory factor analysis (EFA), is a statistical method that seeks to identify groupings of items from an assessment within correlation space based on the students' response vectors. Since this analysis groups items together based on correlations, the correlation matrix of the items (in this case the individual response options for each question) of the assessment must be calculated. This correlation matrix is calculated using the method as explained in the previous section. From this correlation matrix EFA will attempt to create a reduced factor model that adequately recreates the correlation matrix from which it originated. The generated factor structure will place the items onto a limited number of vectors, called factors, by identifying the item's specific loading values on each factor. These item loading values are proportional to the amount of variance that is explained for each item by the factor being examined. Within the tables presented in this study all item loadings below 0.29 were not presented. To find these loading values, the principle axis factoring method was used along with a "oblmin" rotation of the factors using the $R$ function "fa" [18].

There are many methods possible that will generate the factors for EFA, however most of them require the researcher to identify the number of factors that will be used in the analysis. Currently, there is no completely agreed upon methodology for the identification of the appropriate number of factors to retain for EFA. A rather off-the-cuff method to find a suggestion for the number of factors is the scree plot test [19]. This test takes the correlation matrix being considered and plots, in decreasing order, the eigenvalues of the matrix, which can be seen for both the pre- and post-test in Figs. 1 and 2, respectively. From the scree plot, the index where the concavity of the points changes is an indication of the recommended number of factors. Similarly, where there is a large difference between the one eigenvalue and the next is another suggestion for the number of factors to be used for the EFA model. From the pretest scree plot (Fig. 1) it can be seen that 6 factors are recommended for the extraction. With the post-test data, it is more difficult to identify the recommended number of factors, but from the full scree plot (Fig. 2) it appears to also be at 6 factors. Considering the zoomed-in scree plot, it can be seen that either 4 or 6 factors is the suggested number for the post-test when using the scree plot test.

A more sophisticated method for identifying the recommended number of factors for EFA is parallel analysis (PA). PA compares the eigenvalues of the sample's correlation matrix to a baseline correlation matrix. The baseline is generated by creating a matrix of the same size as the original sample, filling it with random normal values (mean $=0$, standard deviation $=1$ ), grading the baseline sample in the same manner as the original sample, and 


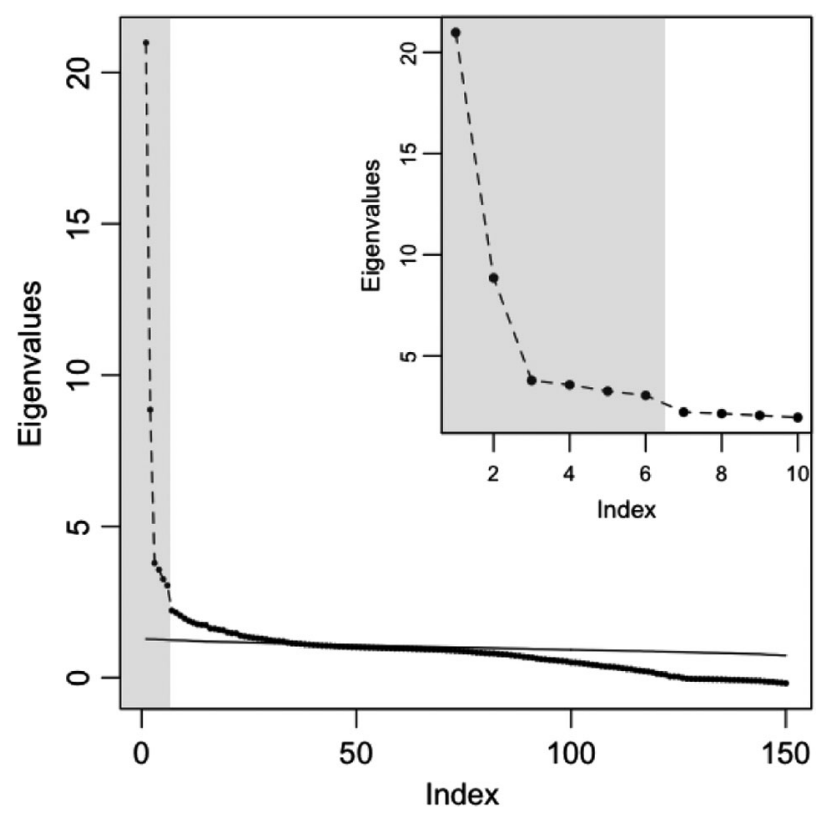

FIG. 1. A scree plot for the pre-instruction sample, where the gray box encloses the first 6 eigenvalues. The inset plot is an enlarged plot of the first 10 eigenvalues. The solid, black line represents the eigenvalues of a baseline sample of the same size as the original sample, with the intersection with the sample's eigenvalues located after index 37.

calculating the resulting correlation matrix. The eigenvalues of this matrix approximate random guessing noise of the sample being studied. Any eigenvalues of the sample that fall below the baseline are said to be below the noise

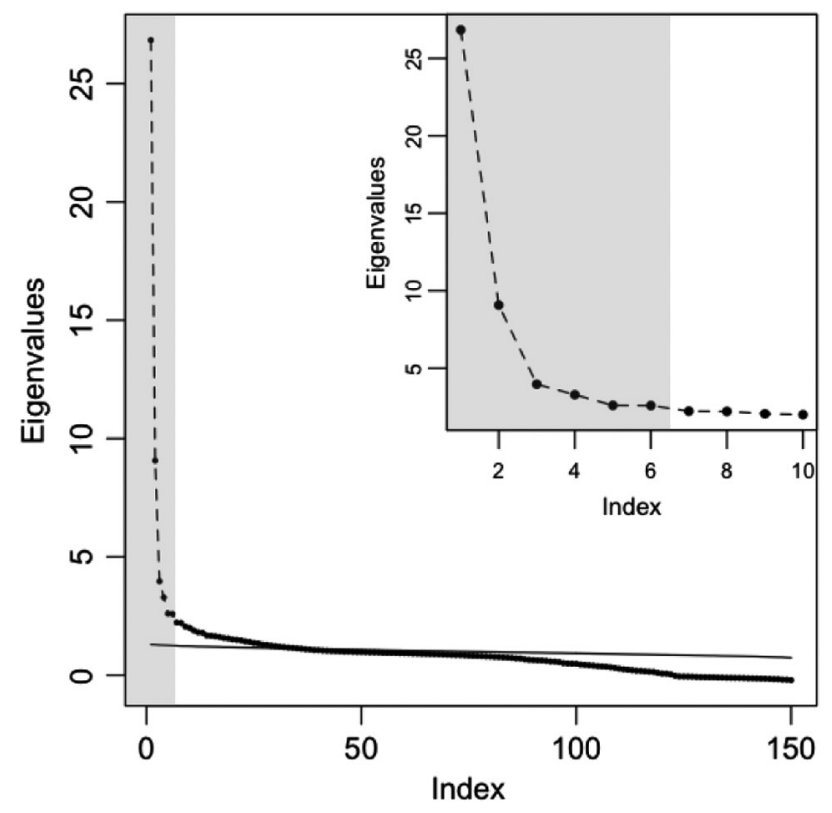

FIG. 2. A scree plot for the postinstruction sample, where the gray box encloses the first 6 eigenvalues. The inset plot is an enlarged plot of the first 10 eigenvalues. The solid, black line represents the eigenvalues of a baseline sample of the same size as the original sample. The intersection is located after index 36 . level and cannot be extracted. Thus, the number of eigenvalues above the baseline indicates the maximum number of factors that can be extracted. Using this method the pretest can have up to 37 factors, and the post-test can have up to 36 .

To help identify the number of factors to be extracted from a correlation matrix, a range of models can be constructed using different numbers of factors and then compared to one another. For instance, when advancing from a two-factor model to a six-factor model using the pretest correlation matrix, significant changes to the factor structure were found as the number of factors were increased. Models constructed using 6 or 7 factors both were equally informative, however, the extra factor in the seven-factor model combined a set of distractors with no correct responses. This analysis uses the correct responses that appear on the same factor as distractors to help identify the Newtonian conception being targeted by the coloading distractors. For factors that contain no correct responses, identifying the students' misconceptions becomes difficult since there is no reference to the proper conception the students are being pulled away from. Additionally, models using more than 7 factors only contain factors with only 2-3 options. These do not constitute a coherent worldview, and were not helpful in trying to understand student misconceptions. Between the six-factor model and the seven-factor model, the six-factor model contained fewer miscellaneous appearing factors and, as a result, was used for the analysis.

Similarly for the post-test, models using 2 to 6 factors result in significant changes to the factor structure, and models using more than 6 factors generate more miscellaneous factors that only contain distractors. Considering what has been discussed, it was decided that 6 factors for the post-test data is the most logical for this analysis.

\section{Hierarchical cluster dendrogram analysis}

To further explore the relationship between the Newtonian conceptions and their related distractors requires identifying subfactor structure for each of the factors found using EFA. This was done using hierarchical cluster analysis. This analysis takes a distance, known as the dissimilarity, between items and forms item clusters. In the case of this study the dissimilarity was taken to be one minus the correlation between the items present on the factor being considered. These clusters are formed by first placing each of the items into their own individual clusters, then taking the two clusters that have the smallest dissimilarity and combining then into one larger cluster. This merger of the most similar clusters is sequentially done until there is only one cluster remaining. An example of a dendrogram plot generated from this analysis can be found in Fig. 3 .

From the dendrograms that get generated, groups that are highly dissimilar can be identified. For instance, in Fig. 3 two distinct groupings can be seen within the dendrogram. 


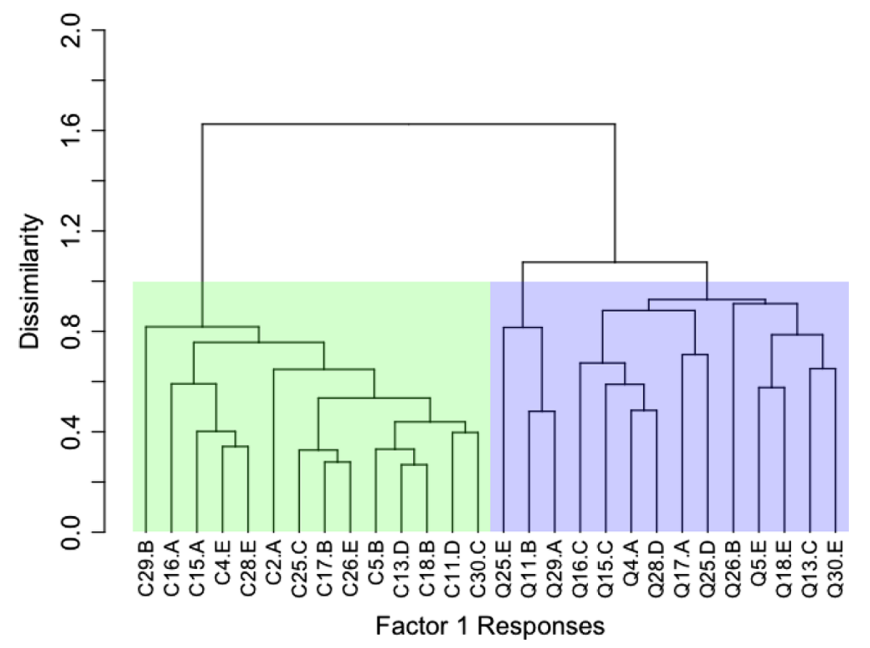

FIG. 3. A hierarchical cluster analysis for the first factor extracted from the pre-instruction response option factor analysis. The top of the shaded rectangles are set at a dissimilarity of 1 , where dissimilarity $=1-$ correlation. Three distinct groupings form-one is made up of correct responses (green) and the other two are groupings of distractors (red).

These groups are linked at a dissimilarity of about 1.6, which means the two groups have a correlation of about -0.6 . So, students tended to choose from one group or the other, but generally not from both simultaneously. As can be seen in Fig. 3, one group is made up of only correct responses and the other is a collection of distractors that pull students away from the correct responses. This is the first step in constituting a coherent alternative worldview held by the students at the time of the assessment. The meaning of this alternative worldview can be discovered by considering the correct responses and the distractors together, as well as the distractor taxonomy presented with the FCI.

\section{Item response curves and item response difference curves}

Item response curves (IRCs) were first presented in 2006 by Morris et al. [12] and were first completely applied to a physics conceptual survey (specifically the FCI) in 2012 in Ref. [6]. Since then, IRCs have been used on the force and motion conceptual survey to identify differences in thinking between Japanese and American students [20].

IRCs are simplified versions of the item characteristics curves found in nominal item response theory [21]. Essentially, these are simple constructions that plot the response percentage of selection options versus the total scores achieved by the students. These plots reveal qualitative patterns within the student responses as a function of the students' abilities (i.e., their total score on the assessment). Taking the pre- and post-test IRCs into consideration reveals the changes in the response options selected by the students over the course of instruction.
To better identify differences in response selections by the students from before to after instruction, slightly modified IRCs were constructed. Specifically, differences in the response selections for students as a function of their pretest scores were found using an IRC-like construction procedure. To build item response difference curves (IRDCs) the students are first binned via the total score achieved on the pretest. From there, the response percentage for each response option within a question are calculated for both pre- and post-test results, all the while leaving the students in their pretest total score bins. Then, the difference between the pre- and post-test response percentages are taken for each option, resulting in a plot that visually shows how the students change their answers from pre- to post-test as a function of their pretest scores. An example of an IRDC using this construction procedure can be found in Fig. 4, and the plots for all of the items on the FCI can be found in the Appendix in Figs. 12-17.

From these curves it can be seen that options with negative response percentage changes are ones that students selected pre-instruction, but did not select again postinstruction. Positive response percentage changes are options that students switched into from their original pretest selection. Since the FCI was created to specifically target a set of alternative world views, the distractors can be related to their parent misconceptions through a distractor taxonomy given with the FCI which is explained in the following section. Thus, the distractors can be plotted using their alternative world view's meaning, and growth of

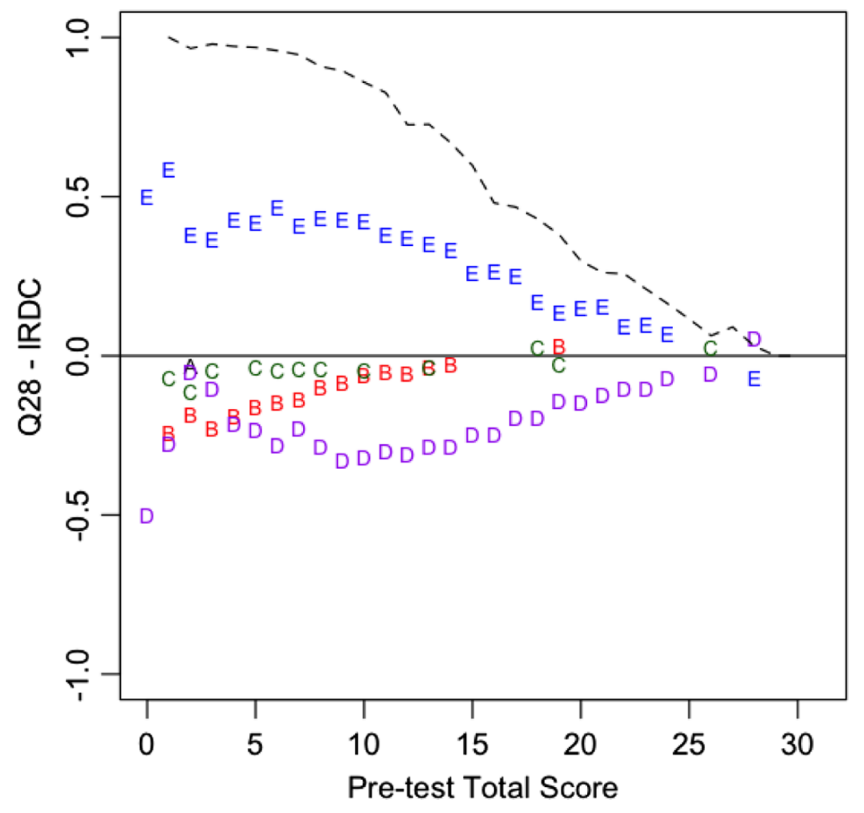

FIG. 4. The item response difference curve for item 28 on the FCI. As can be seen, the students stopped selecting options B, C, and D and mostly changed into option "E," the correct option. This item is about Newton's third law, and distractors B and D are about the active forces and action-reaction pair misconceptions, respectively. 
specific misconceptions can be readily identified (see the third column of Figs. 12-17). Further, the maximum possible growth is plotted as a dashed line over top of the IRDCs for every item to give a feel for how much the students improved as a function of their pretest total scores on each item.

It should be noted that these plots only help to identify the distractors that students moved away from, or into, and do not reveal distractors that remained unaffected after instruction. These resilient distractors will need to be identified by considering the pretest IRCs and the IRDCs for each item to find the distractors whose response percentages that did not change as a result of instruction (see question 21's IRDC in Fig. 16). This is the qualitative method used in this study to identify which misconceptions, according to the FCI, were removed, left untouched, and/or were learned from pre- to postinstruction.

\section{E. Misconception taxonomy}

The coding for the distractor options presented in Table XI, located in the Appendix, is a result of the taxonomy of the distractors given by the creators of the FCI in Ref. [1]. The original taxonomy was for the 1992 version of the FCI and was justified through student interviews performed prior to its construction [22]. However, due to the modifications that were made to the FCI in 1995, the codings in Ref. [1] had to be updated. Since most of the questions remained unchanged, only the new or modified questions' codings were completed without consultation of the original taxonomy. These questions were coded by considering the wording of the unchanged questions, the descriptions of the alternative world views, and the authors' discretion. Thus, an updated distractor taxonomy for the 1995 version of the FCI can be found in Table XI, located in the Appendix.

Upon inspection of the specific misconceptions detailed in the distractor taxonomy, see Table XI, it becomes clear that many of the misconceptions actually pertain to the same world view applied to slightly different situations or in slightly different manners. For example, from the distractor taxonomy in Table XI, I1 (impetus supplied by a "hit") could be a consequence of the idea that force is proportional to velocity. This kind of thought process from a student taking the FCI could potentially cause a link between the I1 and AF1 (motion implies active force) misconceptions. Thus, when the correlations for these options are examined it should not be surprising to find factors that contain a mixture of these highly specific misconception classifications. Without interview data, the explicit cause of these correlations cannot be identified and an investigation of this nature is suggested by the authors for a future extension of this analysis. Consequently, when interpreting the meaning behind factors found in this study a suggestion for the overall misconception that ties the distractors together will be offered with the understanding that there could be many other interpretations that are equally as valid and logical.

The qualitative interpretations for the factors found through this analysis will be derived using one of two different approaches. The distractor taxonomy supplied with the FCI will be used to supply an interpretation for the factors whose displayed distractors all contain the same misconception. In the event some factors do not contain distractors of the same classification, the best loading distractors' content in conjunction with the distractor taxonomy will be used to give these factors their interpretations. The use of each method will be explicitly commented on in the discussion of the factors themselves, see Sec. IV.

\section{RESULTS AND DISCUSSION}

The following is a discussion of the factor analysis results for the pre- and post-test matched data. The factors that are not miscellaneous in nature will be discussed in each of their own subsections, and the miscellaneous factors for the pre- and post-test will be discussed at the end of their own respective sections. Readers are encouraged to have a copy of the FCI with them while going through these sections, as it will more readily enable them to read the set of distractors and correct responses that grouped together within each factor. The discussion of each factor's qualitative meaning will be kept relatively succinct for brevity.

\section{A. Pre-Instruction}

Of the pretest factors, factors 1,3 , and 5 all contain coherent alternative world views held by the students, and are discussed in some detail in the following sections. The other factors are presented, but are concluded to be either miscellaneous groupings of items, student testing strategies, or curious misconception relations.

\section{Factor 1}

The first factor found in the factor analysis of the preinstruction data can be found in Table II and the hierarchical clustering dendrogram is presented in Fig. 3. From the item loading values it can be seen that there are two groups that form. The items with negative loadings are the correct Newtonian responses while the positively loaded items are distractors. The dendrogram shows that the correct responses and the distractors create two distinctly different groups that are anticorrelated with one another, the distractors breaking up into two subsequent groupings of their own.

Of the correct responses, multiple Newtonian concepts appear to be grouped within this factor probing Newton's first and second laws (17.B, 25.C, and 26.E), Newton's third law (4.E, 15.A, 16.A, and 28.E), and force identification (5.B, 11.D, 13.D, 18.B, 29.B, and 30.C). 
TABLE II. Item loading values for pretest factor 1. All loadings above 0.290 were retained in the factor. All of the options with negative loadings are correct responses on the FCI, and the items with positive loadings are all distractors.

\begin{tabular}{lrrrrrrc}
\hline \hline $\mathrm{Q}$ & $\mathrm{L}$ & $\mathrm{Q}$ & $\mathrm{L}$ & $\mathrm{Q}$ & $\mathrm{L}$ & $\mathrm{Q}$ & $\mathrm{L}$ \\
\hline 17.B & -0.775 & 15.A & -0.612 & 4.A & 0.692 & 25.D & 0.406 \\
26.E & -0.739 & 13.D & -0.606 & 28.D & 0.573 & $11 . \mathrm{B}$ & 0.376 \\
25.C & -0.714 & $11 . \mathrm{D}$ & -0.563 & $16 . \mathrm{C}$ & 0.516 & $5 . \mathrm{E}$ & 0.375 \\
4.E & -0.707 & $30 . \mathrm{C}$ & -0.497 & $30 . \mathrm{E}$ & 0.513 & $29 . \mathrm{A}$ & 0.323 \\
28.E & -0.685 & $16 . \mathrm{A}$ & -0.463 & $15 . \mathrm{C}$ & 0.492 & $17 . \mathrm{A}$ & 0.316 \\
18.B & -0.664 & 2.A & -0.421 & 13.C & 0.464 & 26.B & 0.300 \\
5.B & -0.615 & 29.B & -0.346 & 18.E & 0.422 & 25.E & 0.297 \\
\hline \hline
\end{tabular}

Considering the distractors contained in this factor, utilizing the taxonomy provided for the FCI and the wording of the options themselves, two major misconceptions come to light: those being an active agent misconception (4.A, 15.C, 16.C, 17.A, 25.D, and 28.D) and an active force misconception (5.E, 11.B, 13.C, 18.E, 25.E, 26.B, and 30.E).

The presence of the force identification questions appearing with Newton's laws is curious and worth examining whether or not it makes sense for these items to all exist in one grouping. The force identification distractors on this factor are all either missing a force (e.g., a normal force), identify an improper centripetal force, and/or more commonly have a force in the direction of motion of the object being considered. This force in the direction of motion may be a consequence of a active force misconception. So, although the force identification questions appear out of place from a Newtonian content point of view, their associated distractors do fit in with the other distractors on this factor.

Factor 1 appears to contain the active agent and active force misconceptions. These have been found in student response data for past FCI postinstruction results $[10,11,13]$. Identifying these misconceptions in pretest student responses is encouraging, supporting the idea that these misconceptions are self-discovered prior to instruction and are not likely learned through instruction $[22,23]$.

\section{Factor 3}

The correct responses contained in Factor 3, the items with negative loading values in Table III, pertain to path identification and their paired speed identification problems (6.B, 8.B, 10.A, 12.B, 23.B, and 24.A), as well as questions that probe other kinematic characteristics of motion in a uniform gravitational acceleration (1.C and 13.D). Considering the majority of the correct responses contained on this factor, items 1.C and 13.D could be thought of as acceleration identification problems.

The distractors for this factor split into two groups that are anticorrelated to one another, but are both still
TABLE III. Item loading values for pretest factor 3. All loadings above 0.290 were retained in the factor. Of the options with negative loadings all but three are correct responses (21.C, 12.A, 14.C). The items with positive loadings are all distractors.

\begin{tabular}{lcccrcrc}
\hline \hline $\mathrm{Q}$ & $\mathrm{L}$ & $\mathrm{Q}$ & $\mathrm{L}$ & $\mathrm{Q}$ & $\mathrm{L}$ & $\mathrm{Q}$ & $\mathrm{L}$ \\
\hline 10.A & -0.457 & $12 . \mathrm{B}$ & -0.302 & 6.A & 0.505 & 24.E & 0.343 \\
23.B & -0.453 & $13 . \mathrm{D}$ & -0.301 & 10.D & 0.466 & 1.D & 0.341 \\
6.B & -0.429 & & & $14 . \mathrm{A}$ & 0.459 & $12 . \mathrm{D}$ & 0.316 \\
24.A & -0.378 & $21 . \mathrm{C}$ & -0.410 & $7 . \mathrm{A}$ & 0.439 & $13 . \mathrm{B}$ & 0.311 \\
8.B & -0.353 & $12 . \mathrm{A}$ & -0.349 & 8.D & 0.351 & & \\
$1 . \mathrm{C}$ & -0.327 & $14 . \mathrm{C}$ & -0.338 & 23.D & 0.346 & & \\
\hline \hline
\end{tabular}

negatively correlated to the correct answers (see Fig. 5). The group of distractors that is more anticorrelated to the correct responses (1.D, 6.A, 7.A, 8.D, 10.D, 12.D, 13.B, 14.A, 23.D, and 24.E) is mostly coded as the impetus misconception from the FCI distractor taxonomy, Table XI. Specifically, the misconception that impetus is a property of objects that can be retained from a hit, dissipated, built up, etc. The only distractors that do not probe impetus are items 1.D and 14.A, which are coded as G3 and R1 as in Table XI. An attempt was made to give these items an impetus interpretation, however one could not be found.

The other set of distractors contains three items (12.A, 14.C, and 21.C) and do not appear to be about impetus as a property of objects. These items all are coded as CI2, which probes the misconception that the motion of an object is in the same direction as the total composition of the forces (i.e., the net force) currently acting on the object. For example, 12.A and 14.C are from path identification problems for two-dimensional projectile motion where

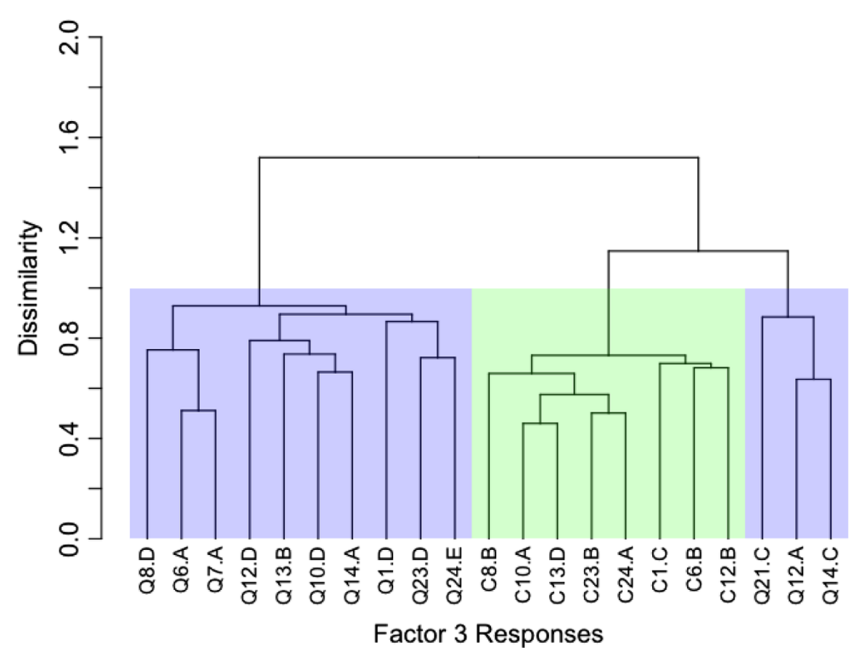

FIG. 5. A hierarchical cluster analysis for the third factor extracted from the pre-instruction response option factor analysis. The top of the shaded rectangles are set at a dissimilarity of 1, where dissimilarity $=1-$ correlation. Three distinct groupings form-one is made up of correct responses (green) and the other two are groupings of distractors (red). 
the selected paths are straight lines directed from the point of launch to the point of landing. This path, according to the misconception taxonomy, is selected since the force down due to gravity and a force to the right, apparently supplied by the cannon or the plane, creates a net force down and to the right. Thus, the ball in each problem will take a straight line path as dictated by the direction of this net force.

This factor contains two anticorrelated alternative world views: impetus as a property of an object, and that motion of an object is always in the same direction as the net force acting on the object. The impetus misconception has been seen in postinstruction student responses in previous analyses (see Refs. [10,11,13]). However, the net force dictating motion misconception has not been seen in the world view statistical studies found in the literature as of the time of this study.

\section{Factor 5}

Similar to the third pre-instruction factor, the fifth factor contains path identification and/or speed identification questions for accelerating systems (9.E, 14.D, 19.E, 21.E, 22.B, and 27.C). The only exception being item 23.B, which is a path identification question for a rocket that has just finished accelerating. All of the correct answers negatively load onto this factor (see Table IV) and are highly anti-correlated to the distractors that also appear on this factor (see Fig. 6).

The distractors on this factor appear to form two uncorrelated groupings. The larger of the two groups (8.A, 9.B, 14.B, 21.B, 23.C, and 27.A) mostly contains distractors that probe the CI3 misconception, see Table XI. This misconception is the idea that the last force to act on an object fully determines the motion of the object. A good example of this is item 21.B, where a rocket is drifting through space to the right and turns on its thrusters directed up the page. The students incorrectly think the resulting motion of the rocket will only be up the page, forgetting entirely about the initial rightward drift. The other two distractors (14.B and 27.A) from this group are coded as probing different misconceptions from the rest of the distractors. However, the situations proposed and the text for each of these distractors readily lends themselves to the same interpretation as the other distractors on this factor. For instance, item 14.B is about a ball falling out of a plane,

TABLE IV. Item loading values for pretest factor 5. All loadings above 0.290 were retained in the factor. All of the options with negative loadings are correct responses and the items with positive loadings are all distractors.

\begin{tabular}{lrrrrccc}
\hline \hline $\mathrm{Q}$ & $\mathrm{L}$ & $\mathrm{Q}$ & \multicolumn{1}{c}{$\mathrm{L}$} & \multicolumn{1}{c}{$\mathrm{Q}$} & $\mathrm{L}$ & $\mathrm{Q}$ & $\mathrm{L}$ \\
\hline 21.E & -0.420 & 23.B & -0.342 & $21 . \mathrm{B}$ & 0.566 & 23.C & 0.399 \\
14.D & -0.415 & $27 . \mathrm{C}$ & -0.316 & 9.B & 0.457 & 27.A & 0.362 \\
9.E & -0.383 & $19 . \mathrm{E}$ & -0.294 & 22.A & 0.453 & 26.A & 0.359 \\
22.B & -0.350 & $8 . \mathrm{A}$ & 0.595 & $14 . \mathrm{B}$ & 0.437 & 23.A & 0.297 \\
\hline \hline
\end{tabular}

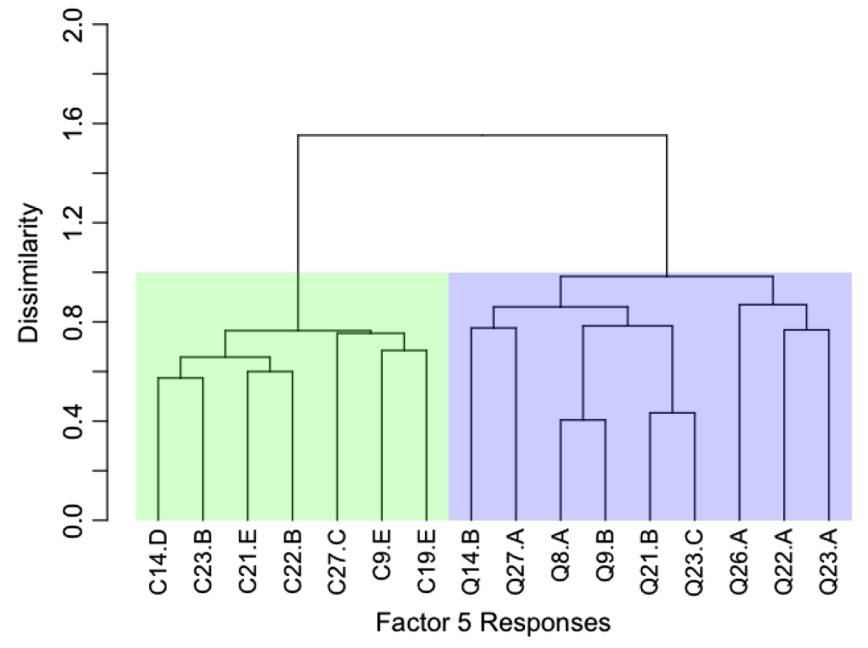

FIG. 6. A hierarchical cluster analysis for the fifth factor extracted from the pre-instruction response option factor analysis. The top of the shaded rectangles are set at a dissimilarity of 1 , where dissimilarity $=1-$ correlation. Two distinct groupings form-one is made up of correct responses (green) and the other is a grouping of distractors (red).

where the path identified in this response is one that falls straight down forgetting about the initial rightward motion of the ball while in the plane. In this context, this item is identical to 21.B and can be assumed to probe the same misconception. In item 27.A, a woman is initially sliding on a box along the floor. She suddenly stops pushing on the box and this distractor claims that the box will immediately come to a stop. This could be because the force in the direction of motion ceased and only friction is acting on the box as a result. Since friction will not cause the box to move backwards, the box will simply stop in place. Using this kind of logic, it can be seen how the students could place all of these distractors into the same alternative world view framework.

The smaller distractor group (22.A, 23.A, and 26.A) appears to investigate the misconceptions that the net force acting on an object is proportional to the object's velocity. The two misconceptions contained in this one factor are similar in nature but are situationally different. The combining of these misconceptions into one factor can be explained by considering the following logic that may be held by the students. For instance, the idea that the last force to act on an object fully determines the current motion of the object could be explained by a student thinking that the velocity of an object is directly proportional to the force being applied to the object. So, when a new force is applied to the object, the object's motion changes in an abrupt manner. Following this logic, the three distractors in the smaller grouping could be explained by a more novicelike thinking compared to the larger distractor grouping, where the students fail to realize that a net force in the direction of motion on an object will cause it to increase its speed, or vice versa. 
TABLE V. Item loading values for pretest factors 2, 4, and 6. All loadings above 0.290 were retained in the factor. All of the options on these three factors are distractors.

\begin{tabular}{|c|c|c|c|c|c|c|c|}
\hline \multicolumn{4}{|c|}{ Pre Factor 2} & \multicolumn{2}{|c|}{ Pre Factor 4} & \multicolumn{2}{|c|}{ Pre Factor 6} \\
\hline Q & $\mathrm{L}$ & Q & $\mathrm{L}$ & Q & $\mathrm{L}$ & Q & $\mathrm{L}$ \\
\hline 29.C & 0.578 & 4.B & 0.414 & 15.E & 0.919 & 29.A & 0.509 \\
\hline 4.C & 0.548 & 6.D & 0.401 & 14.E & 0.883 & 15.D & 0.381 \\
\hline 17.C & 0.542 & 25.A & 0.387 & 13.E & 0.777 & 28.B & 0.359 \\
\hline 12.A & 0.520 & 10.B & 0.362 & $6 . \mathrm{E}$ & 0.455 & 16.D & 0.313 \\
\hline 24.B & 0.519 & 16.B & 0.356 & 27.E & 0.426 & 18.A & 0.290 \\
\hline 26.C & 0.502 & 19.B & 0.356 & 17.E & 0.412 & 11.C & -0.613 \\
\hline 22.C & 0.499 & 27.D & 0.355 & 16.E & 0.387 & 5.D & -0.319 \\
\hline $8 . \mathrm{C}$ & 0.452 & 28.A & 0.346 & 11.E & 0.365 & & \\
\hline \multirow[t]{4}{*}{ 30.A } & 0.425 & 24.D & 0.336 & 29.E & 0.340 & & \\
\hline & & & & 28.A & 0.327 & & \\
\hline & & & & 2.E & 0.295 & & \\
\hline & & & & 22.E & 0.290 & & \\
\hline
\end{tabular}

From these considerations, the fifth factor for the pretest appears to be uniformly probing the misconception that the total force acting on an object is proportional to the object's resulting vector motion. This alternative world view appeared, in part, in the pretest factor 3's distractors as well.

\section{Factors 2, 4, and 6}

The following factors, item loading values displayed in Table V, all seem to be either miscellaneous in nature or reflect student testing strategies when taking a multiple choice assessment.

Factor 2 contains a mixture of misconception codings that do not form any coherent alternative world views. In fact, all of the items on this factor were selected by less than 5\% of the 19727 student sample. As a result, this factor is assumed to not contain a prominent world view held by the majority of the students in the sample.

Factor 4 appears to recover the test strategy that if a student does not know what the answer is, then they will select option ' $\mathrm{E}$ ' for that question. Of the 12 items on factor 4, 11 of them are option ' $E$ ' from the 'A'- 'E' response options available for the questions on the FCI. When the responses themselves are considered, no coherent alternative world view is apparent as an explanation for the distractor collection. Factors like this have been found in previous studies, thus finding one in this sample is not surprising [10].

Factor 6 does not contain any correct responses, however it does have two anticorrelated groups of distractors, see Fig. 7. Items 5.D and 11.C both are about the misconception that there needs to be a force in the direction of motion even when the object is not changing speed. The other distractors in this factor (15.D, 16.D, 18.A, 28.B, and 29.A) are all missing required forces in one form or another, predominantly the normal force. Note that these sets of distractors are anticorrelated, which may indicate that when

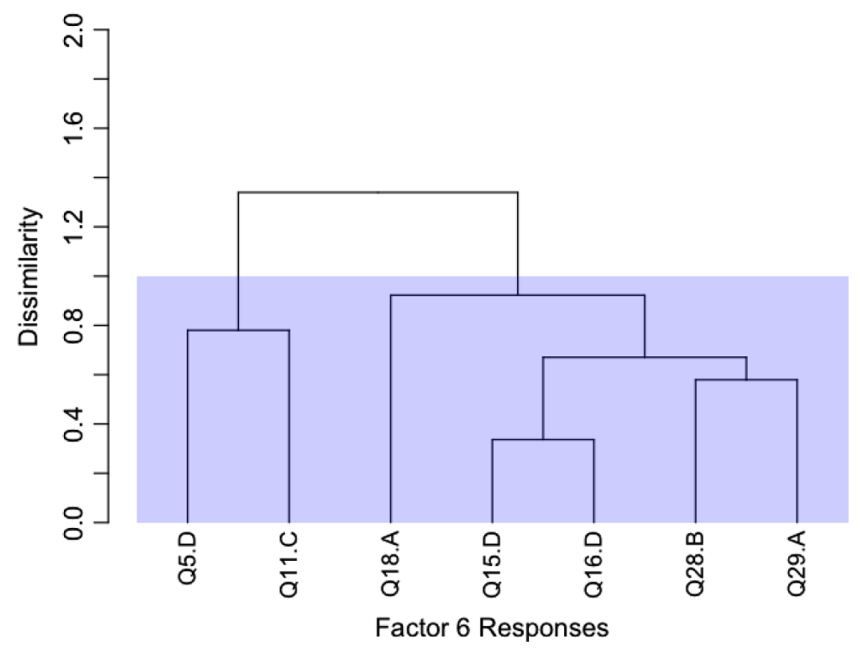

FIG. 7. A hierarchical cluster analysis for the sixth factor extracted from the pre-instruction response option factor analysis. The top of the shaded rectangles are set at a dissimilarity of 1 , where dissimilarity $=1-$ correlation. Two distinct groupings form, both of which are groupings of distractors (red).

some students learn how to properly identify forces (e.g., the normal force) prior to instruction they may inadvertently adopt the misconception that a force is needed in the direction of motion for an object to move.

\section{B. Postinstruction}

Of the post-test factors, factors $2,3,4$, and 5 all contain coherent alternative world views held by the students. Factors 1 and 6 are concluded to be either miscellaneous or reflecting student testing strategies.

\section{Factor 2}

Factor 2 contains 13 correct answers, all with negative loadings in Table VI, which are about force identification (5.B, 11.D, 13.D, 18.B, and 30.C) and Newton's first or second law (2.A, 17.B, 20.D, 21.E, 22.B, 25.C, and 26.E). The distractors within this factor create three distinct groups, two of which probe a similar alternative world view.

TABLE VI. Item loading values for post-test factor 2. All loadings above 0.290 were retained in the factor. Of the options with negative loadings, only one was not a correct response (item 5.A). The items with positive loadings are all distractors.

\begin{tabular}{lrrrrrrr}
\hline \hline $\mathrm{Q}$ & $\mathrm{L}$ & $\mathrm{Q}$ & $\mathrm{L}$ & $\mathrm{Q}$ & $\mathrm{L}$ & $\mathrm{Q}$ & $\mathrm{L}$ \\
\hline 13.D & -0.660 & $22 . \mathrm{B}$ & -0.428 & $11 . \mathrm{C}$ & 0.676 & 22.A & 0.382 \\
26.E & -0.638 & 2.A & -0.328 & 13.C & 0.632 & 5.E & 0.361 \\
18.B & -0.626 & $20 . \mathrm{D}$ & -0.321 & 30.E & 0.603 & $18 . \mathrm{E}$ & 0.355 \\
25.C & -0.575 & $21 . \mathrm{E}$ & -0.320 & 18.D & 0.575 & 20.C & 0.339 \\
17.B & -0.569 & & & 5.D & 0.500 & 26.A & 0.325 \\
5.B & -0.561 & & & 17.A & 0.463 & 26.B & 0.305 \\
30.C & -0.552 & & & 25.D & 0.448 & $13 . \mathrm{B}$ & 0.302 \\
11.D & -0.523 & 5.A & -0.304 & 21.C & 0.419 & $10 . \mathrm{E}$ & 0.299 \\
\hline \hline
\end{tabular}




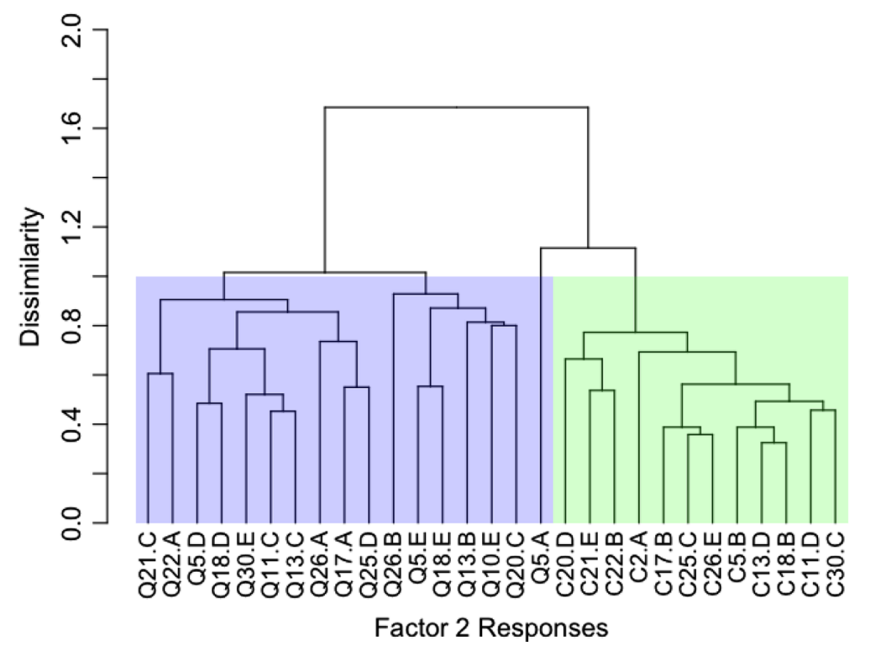

FIG. 8. A hierarchical cluster analysis for the second factor extracted from the postinstruction response option factor analysis. The top of the shaded rectangles is set at a dissimilarity of 1, where dissimilarity $=1-$ correlation. Three distinct groupings form, one is made up of correct responses (green) and the others are groupings of distractors (red).

The two larger groupings of distractors, see Fig. 8, do not contain clear groupings of the misconception codings from Table XI. Considering these codings originated from student "common sense" formed prior to instruction, it is not surprising that they do not form coherent groupings after instruction [22]. When the content of these distractor groupings are considered, it becomes apparent that one is a more novice grouping of distractors compared to the other, with both suffering from the same overarching conceptual issue. The more novice group (5.E, 10.E, 13.B, 18.E, 20.C, and 26.B) contains distractors that are missing needed forces, mistake acceleration for velocity, and/or have a force in the direction of motion when one is not necessary. The other grouping (5.D, 11.C, 13.C, 17.A, 18.D, 21.C, 22.A, 25.D, 26.A, and 30.E) appears to be predominantly contain the misconception that there needs to be a force in the direction of motion even when the speed of the object is not changing. The distractor groupings in this factor are quite mixed and generating an all encompassing explanation for the groups does not seem entirely possible. From the item loadings on this factor the first 7 distractors with the largest loadings contain the force in the direction of motion misconception. Using this as a guide, factor 2 is said to probe the force in the direction of motion misconception in conjunction with other, more minor, issues.

\section{Factor 3}

The correct responses within this factor, all of which have negative loading values in Table VII, are either path identification questions or ask about the motion of objects that have changed speed or are in the process of doing so. The distractors on this factor, similar to factor 2 , do not appear to form a grouping centered on the distractor
TABLE VII. Item loading values for post-test factor 3. All loadings above 0.290 were retained in the factor. All of the options with negative loadings are correct responses and the items with positive loadings are all distractors.

\begin{tabular}{|c|c|c|c|c|c|c|c|}
\hline Q & $\mathrm{L}$ & Q & $\mathrm{L}$ & Q & $\mathrm{L}$ & Q & $\mathrm{L}$ \\
\hline 23.B & -0.458 & 27.C & -0.314 & 23.C & 0.490 & 5.A & 0.358 \\
\hline 14.D & -0.403 & & & $9 . \mathrm{B}$ & 0.481 & 12.E & 0.345 \\
\hline 9.E & -0.377 & & & 14.B & 0.481 & 7.E & 0.337 \\
\hline 21.E & -0.370 & 21.B & 0.717 & 27.A & 0.408 & 26.A & 0.334 \\
\hline $8 . \mathrm{B}$ & -0.325 & 8.A & 0.688 & 22.A & 0.373 & 18.A & 0.298 \\
\hline
\end{tabular}

codings as given by Table XI. So, the wording of the distractors themselves must be considered to give a misconception interpretation. The 6 largest loading distractors (8.A, 9.B, 14.B, 21.B, 23.C, and 27.A) on this factor will be used to help guide the interpretation of the alternative world view being probed. These items pertain to the misconception that the last force that acted on an object dictates the subsequent motion of the object. The items are either coded as being CI3 or R1; see Table XI. Many of the other items investigate the active force misconception (5.A, 18.A, 22.A, and 26.A), which can be summarized by the need for a force that is proportional to the object's velocity.

Two of the distractors in factor 3, as a pair, appear to be uncorrelated to the other distractors on this factor; see Fig. 9. The items both ask about the forces acting on an object in the process of traveling along a circular path. The distractors identified are identical to one another in that they only identify the force due to gravity on the object, and miss the force directed into the center of the circular path. These two items were not among the strongest loading

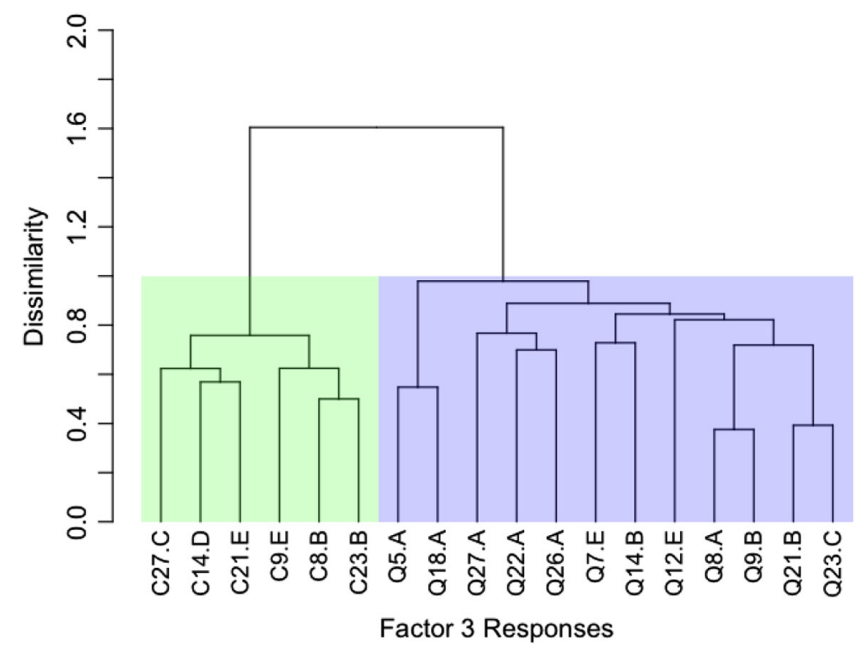

FIG. 9. A hierarchical cluster analysis for the third factor extracted from the postinstruction response option factor analysis. The top of the shaded rectangles are set at a dissimilarity of 1, where dissimilarity $=1-$ correlation. Two distinct groupings form, one is made up of correct responses (green) and the other is a grouping of distractors (red). 
distractors and will not be considered further when giving a qualitative interpretation for this factor.

As a result, factor 3 is thought to be probing two misconceptions that the students have intertwined, the last force acting on an object dictates the subsequent motion of the object and active force.

\section{Factor 4}

All of the correct responses on this factor are from path or speed identification problems for nonaccelerating objects. Four of these items come in pairs (8.B with 10.A and 23.B with 24.A), which ask the students to identify the path taken by a nonaccelerating object, then to comment on how the speed of the object is changing. Looking at the coding for most of the distractors on this factor (the positively loaded items in Table VIII) it can be seen that they almost all are coded as probing the impetus world view, with the exception of items 14.A and 22.E. The wording of these items shows that they could be explained using an impetus world view, particularly that impetus can be dissipated from an object over time.

There are two other distractors that loaded negatively onto this factor, and from Fig. 10 are seen to still be anticorrelated with the Newtonian responses. These items, 14.C and 21.C, contain the misconception that an object's motion is in the direction of the net force acting on the object. This is similar to the misconception probed by factor 3, but these items require superposition of forces to get from the alternative world view probed in factor 3 to the one probed by these items.

Considering the larger distractor grouping of factor 4 , the alternative world view being probed seems to be about impetus as a property of an object being something that can be dissipated, gradually built up or lost, or recovered or built up in a delayed manner. This thinking is similar to how energy operates within objects, and may indicate a poor physical intuition or understanding postinstruction of energy. Without interview data, no conclusions can be made about a possible energy interpretation and it is suggested by the authors as a future study. A distractor grouping like this was also found in Ref. [10], which lends evidence that this actually is a coherent alternative world

TABLE VIII. Item loading values for post-test factor 4. All loadings above 0.290 were retained in the factor. Items 21.C and 14. $\mathrm{C}$ are incorrect responses and the rest of the negatively loaded items are the correct options for their respective questions. The items with positive loadings are all distractors.

\begin{tabular}{lccccccc}
\hline \hline $\mathrm{Q}$ & $\mathrm{L}$ & $\mathrm{Q}$ & $\mathrm{L}$ & $\mathrm{Q}$ & $\mathrm{L}$ & $\mathrm{Q}$ & $\mathrm{L}$ \\
\hline 23.B & -0.373 & 21.C & -0.451 & 7.A & 0.486 & 23.E & 0.374 \\
24.A & -0.370 & $14 . \mathrm{C}$ & -0.422 & 6.A & 0.470 & $14 . \mathrm{A}$ & 0.340 \\
8.B & -0.364 & & & 8.D & 0.442 & 21.A & 0.332 \\
6.B & -0.351 & & & 10.D & 0.430 & 24.C & 0.329 \\
10.A & -0.322 & & & 23.D & 0.380 & 22.E & 0.308 \\
\hline \hline
\end{tabular}

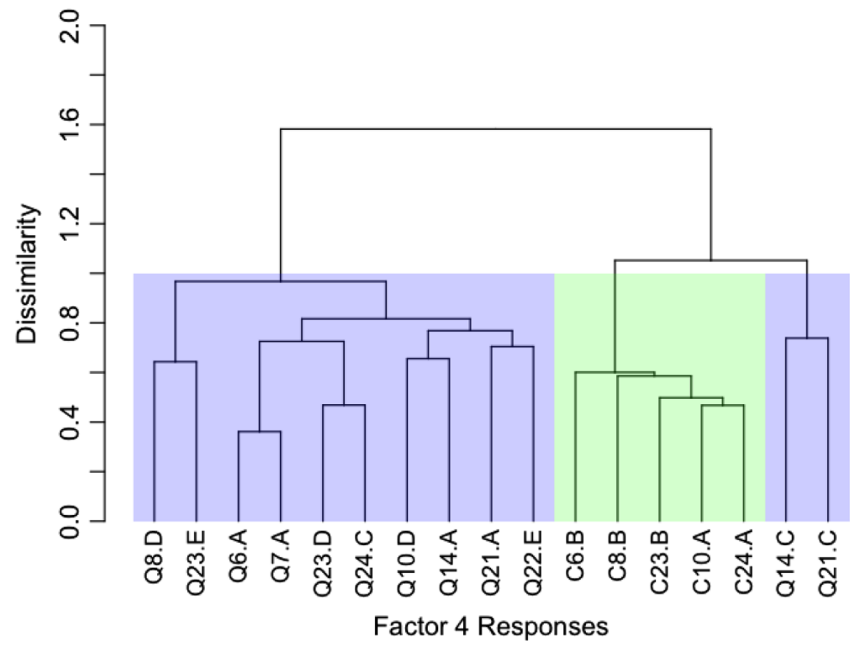

FIG. 10. A hierarchical cluster analysis for the fourth factor extracted from the postinstruction response option factor analysis. The top of the shaded rectangles are set at a dissimilarity of 1, where dissimilarity $=1-$ correlation. Three distinct groupings form, two are made up of correct responses (green) and the other is a grouping of distractors (red).

view still held by students after instruction for the population of students both samples were drawn from.

\section{Factor 5}

The positively loaded items on factor 5, see Table IX and Fig. 11, are all distractors that probe the action-reaction pairs misconceptions. Specifically, the prominent misconception is that the more active agent of two objects pushing on one another will produce the greatest force. The correct responses (4.E, 15.A, and 28.E) are all about Newton's third law, making this factor solely about Newton's third law. The inclusion of item 16.E in this factor is curious considering it is not an action-reaction pair misconception distractor. However, the observation that item 16 does not function properly as a Newton's third law question has been seen in past studies $[7,24,25]$. This lends evidence that question 16 is not seen by the students as a Newton's third law problem, and is instead seen as probing other aspects of dynamics.

TABLE IX. Item loading values for post-test factor 5. All loadings above 0.290 were retained in the factor. Of the options with negative loadings are correct responses item 16.E is an incorrect response and the rest of the negatively loaded items are the correct options for their respective questions. The items with positive loadings are all distractors.

\begin{tabular}{lccccccc}
\hline \hline $\mathrm{Q}$ & $\mathrm{L}$ & $\mathrm{Q}$ & $\mathrm{L}$ & $\mathrm{Q}$ & $\mathrm{L}$ & $\mathrm{Q}$ & $\mathrm{L}$ \\
\hline 15.A & -0.701 & 28.E & -0.587 & 4.A & 0.705 & $15 . \mathrm{C}$ & 0.626 \\
4.E & -0.652 & $16 . \mathrm{E}$ & -0.293 & 28.D & 0.644 & $16 . \mathrm{C}$ & 0.378 \\
\hline \hline
\end{tabular}




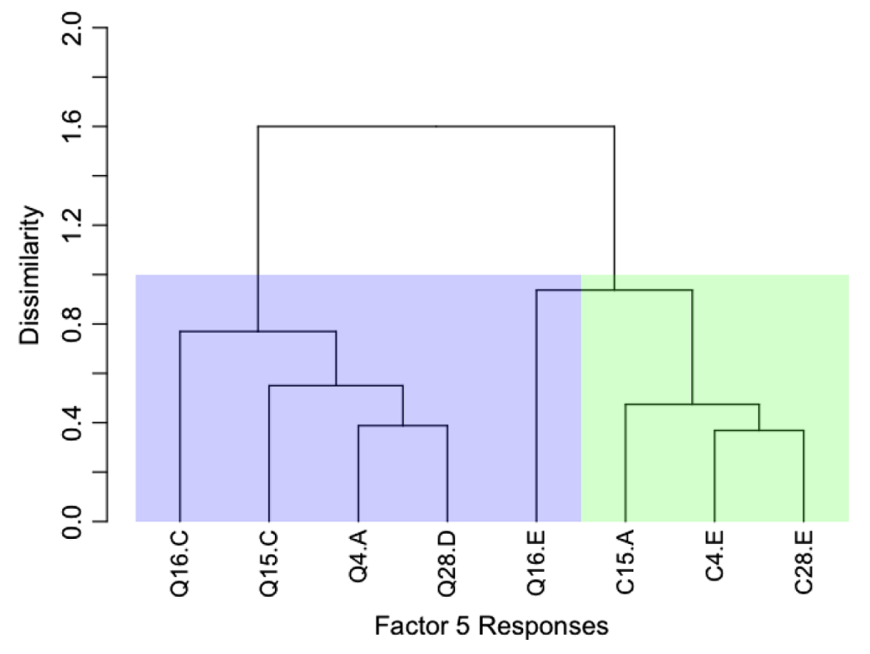

FIG. 11. A hierarchical cluster analysis for the fifth factor extracted from the postinstruction response option factor analysis. The top of the shaded rectangles are set at a dissimilarity of 1, where dissimilarity $=1-$ correlation. Two distinct groupings form, one is made up of correct responses (green) plus a distractor and the other is a grouping of distractors (red).

\section{Factors 1 and 6}

The remaining 2 factors from the generated post-test EFA model do not seem to be probing coherent sets of alternative world views. For instance, factor 6 contains 11 distractors, of which 8 are option ' $E$ ' for their respective questions, see Table X. This factor, similar to factor 4 from the pre-test, seems to recover the student testing strategy that students tend to select option ' $E$ ' if they do not know the answer to the question.

The first factor, which contains 38 individual distractors, looks to be a miscellaneous grouping of items. To support

TABLE X. Item loading values for post-test factors 1 and 6. All loadings above 0.290 were retained in the factor. All of the negatively loading responses are correct options, with the exception of 16.E which is an incorrect response. The items with positive loadings are all distractors.

\begin{tabular}{|c|c|c|c|c|c|c|c|}
\hline \multicolumn{6}{|c|}{ Factor 1} & \multicolumn{2}{|c|}{ Factor 6} \\
\hline Q & $\mathrm{L}$ & Q & $\mathrm{L}$ & Q & $\mathrm{L}$ & Q & $\mathrm{L}$ \\
\hline 16.A & -0.321 & 12.A & 0.446 & $15 . \mathrm{E}$ & 0.354 & 13.E & 0.628 \\
\hline 24.A & -0.302 & 24.B & 0.439 & 30.A & 0.353 & 1.E & 0.509 \\
\hline \multirow[t]{2}{*}{ 19.E } & -0.291 & 19.B & 0.435 & 9.A & 0.339 & 14.E & 0.482 \\
\hline & & 27.D & 0.433 & 2.C & 0.332 & 15.E & 0.427 \\
\hline 29.C & 0.721 & 28.B & 0.428 & 19.C & 0.328 & 29.E & 0.370 \\
\hline 17.C & 0.692 & 28.A & 0.418 & 30.D & 0.322 & 17.D & 0.342 \\
\hline 4.C & 0.611 & 4.D & 0.406 & $18 . \mathrm{C}$ & 0.319 & 6.C & 0.330 \\
\hline 8.C & 0.571 & 6.D & 0.406 & 6.E & 0.310 & 2.E & 0.327 \\
\hline 26.C & 0.570 & 10.B & 0.405 & 20.B & 0.307 & 6.E & 0.314 \\
\hline 22.C & 0.502 & 16.D & 0.391 & 29.A & 0.304 & 4.C & 0.306 \\
\hline 24.D & 0.473 & 25.A & 0.388 & 18.A & 0.301 & 17.E & 0.299 \\
\hline 16.B & 0.470 & 25.B & 0.387 & 15.B & 0.298 & & \\
\hline 27.E & 0.465 & $28 . C$ & 0.378 & 21.A & 0.294 & & \\
\hline 15.D & 0.462 & 4.B & 0.359 & 13.A & 0.291 & & \\
\hline
\end{tabular}

this claim the first 6 best loading distractors are all option ' $\mathrm{C}$ ' and do not probe similar misconceptions. Further, the correct responses contained on this factor, the negatively loaded items, also do not pertain to the same Newtonian conceptions. Consequentially, this factor is concluded to be a miscellaneous grouping of items produced from the EFA factor construction procedures which requires all of the items given in the correlation matrix to load onto a factor.

\section{Comparing the pre- and post-test EFA results}

Comparing the pre-test EFA results to the post-test EFA results reveals some similarities in the alternative world views held by the students in the sample from pre- to posttest. For instance, the first factor for the pre-test and the second and fifth factor for the post-test hold similar conceptions to one another. The second factor on the post-test contains the alternative world view that motion requires a force even if the object is moving with a constant velocity, and the fifth factor is about action-reaction pair misconceptions. These together form the same set of misconceptions held by the first factor of the pretest student responses. The splitting up of the first pretest factor into two post-test factors which pertain to Newton's third law and a Newton's first and second law could be due to an improvement of the Table Newtonian conceptual understanding. Because of the refined Newtonian thinking by some of the students, the action-reaction misconception was able to be extracted from the force and implies motion misconception originally of the same pretest factor.

As discussed, the third factor of the pretest and the fourth factor of the post-test contained many distractors that probed the impetus misconception, specifically that impetus is a property of an object that can be dissipated, built up, etc. The distractor taxonomy in Table XI shows that almost all of the distractors on these factors are probing impetus as a property of an object in one manner or another. Of all the alternative world view factors found for the pretest and post-test, this misconception is the one that remains the most consistent with the distractor taxonomy given with the FCI. This may be an indication that this alternative world view is "rigid," and is therefore difficult to unlearn for introductory physics students [1,22,23].

The distractors contained on the pretest's fifth and the post-test's third factors contain very similar distracting items. Thus, it not surprising that they were found to probe the same misconceptions. Specifically, these factors probe the misconception that the motion of an object is dictated by the last individual force, or the current net force, acting on the object. This misconception is particularly novicelike and may be a misconception that many instructors do not think about when constructing their curriculum. The prevalence of this misconception from pre- to post-test is interesting, and some level of care is suggested in the instruction process to address this alternative world view specifically. 
Between the pretest and post-test results, the pretest seems to have more coherent distractor groupings and less coherent Newtonian groupings, whereas the post-test results contain more coherent Newtonian groupings and distractor groupings that are harder to identify a single, explanatory misconception. This could be an indication that the students have exchanged part of their alternative world views in favor of a more Newtonian world view as a result of instruction. However, since all of the students are not yet completely Newtonian thinkers, they still have a tendency to select responses based on their old world views when they are faced with a situation where they do not know how to apply proper Newtonian thinking. If this is so, it would not be surprising to find that the pretest and post-test factors are probing similar alternative world views. From this analysis it does not appear as if the post-test alternative world views are being taught during instruction, rather they are remnants of the world views students held prior to instruction. To further investigate this claim, the IRDCs will be considered in the next section to see which, if any, alternative world views were promoted over the course of instruction.

\section{Item response difference curves}

Figures 12-17, in the Appendix display the pretest IRCs, the IRDCs using the option letters as the plotting symbols, and an enlarged version of the IRDCs using the distractor taxonomy from Table XI as the plotting symbols (from left to right in the first three columns of the figures mentioned). The plot in the rightmost column of these figures displays the percent change of the option response rate for each question as a function of the pretest total score achieved by the students. All of these plots contain an abundance of information for every question on this assessment, and an entire future study could be dedicated to dissecting them to reveal information about each question and their distractors. For example, the middle two columns show which options the students, grouped by pretest total scores, selected with less frequency from pre- to post-test, indicated by a negative value on these plots. Additionally, these plots help to show that for most questions the students stop selecting a distracting option, and change to the correct option, indicated by a positive value on these plots. For brevity, only the most prominent features of these plots will be commented on for questions that behaved in interesting manners.

Upon inspection, a number of questions had little change in the response selections made by the students over the course of instruction. These are questions 9, 14, 19, 20, 21, and 27. Question 9 describes a hockey puck initially sliding to the right, then given an instantaneous kick up the page, and asks about the resulting speed in relation to the initial speed to the right and the speed up the page given by the kick. This question seems out of place with the other questions on the FCI, given that its solution requires a student to know a mathematical definition (i.e., magnitude of a vector) as opposed to a conceptual argument, which can be done for all of the other items on the FCI. Considering the initial poor performance of the students on this question during the pretest, and the lack of change from the wrong response " $C$," it can be inferred that students still struggle with vector addition and vector magnitudes after instruction. Specifically, response C says that the resulting speed of the puck should be equal to the sum of the initial speed and the speed from the kick, which indicates that students still lack understanding about the vector nature of velocity.

Question 14 is a path identification problem for a ball being dropped from a plane. There is relatively good movement away from incorrect response "A," but less movement from incorrect response "B." Response B is the path that falls straight down from the point the ball was dropped and probes the misconception that motion is determined by the last force to act on the object, which was seen to exist before and after instruction.

Questions 19 and 20 are strobe-diagram questions that investigate students' understanding of how to infer speed and acceleration from these kinds of plots. Specifically, for question 19 students still seem to mistake the position of objects being equal as their current speeds being equal (response "D") and, similarly, for question 20 with a larger speed being interpreted as a larger acceleration [26,27].

Of all the questions on the FCI question 21 showed a significant promotion of a misconception from pre- to postinstruction. This question is about a rocket drifting to the right that turns on its thrusters, pushing the rocket up the page, and asks the students to identify the correct path the rocket will take as a result. This question shows equal growth of the correct answer and incorrect response $\mathrm{C}$ for students who scored a $50 \%$ or below on the FCI pre-instruction. This response, according to the distractor taxonomy, pertains to the idea that the combined force on the object determines the resulting motion of the object, even though there was never a force acting on the rocket to the right. The drastic difference in the behavior of this item to the other items that probe the same misconception group (questions 6, 7, 8, 9 12,14, 17, and 23) leads the authors to suspect that this behavior may be connected more to the question malfunctioning, and may not reflect novicelike growth in the students' conceptions. This question has been commented on in a previous study where the wording was called into question as a possible explanation for its seemingly poor performance [28].

Lastly, question 27 is about a box that was being accelerated to the right while sliding along the ground. The woman pushing on the box suddenly stops and the students are asked about the subsequent motion of the box. There seems to be minimal motion away from incorrect responses $\mathrm{A}$ and $\mathrm{B}$, which probe the ideas of the last force acting on an object dictates motion of the object and that 
impetus can have a delayed dissipation, respectively. Both of these misconceptions were seen in the pre- and post-test alternative world views, and this question demonstrates how hard it may be for students to overcome these misconceptions.

The remainder of the questions contained "good" growth of the correct response, however some questions show that the students are not always moving from an incorrect response to a correct response. This can be seen in questions $2,5,11,18,22,26$, and 30 , where one or two distractors are promoted from pre- to post-test. Considering the taxonomy of these distractors, it can be seen that the majority (questions 5, 18, 22, 26, and 30) of the promoted distractors are about the active force misconception. Question 2 saw growth of the "heavier objects fall faster" misconception and question 11 saw growth in the impetus supplied by a hit misconception.

The results of this qualitative analysis support that the force determining motion and active force misconceptions are failing to be completely fixed over the course of instruction. Specifically, the large number of questions that contained growth of the active force misconception show that this alternative world view deserves special attention during instruction. This has been recommended previously by Scott and Schumayer [10].

\section{LIMITATIONS}

The data used in this study were from a mixture of algebra- and calculus-based introductory mechanics physics classes, thus the factors found may change when considering specific levels of mathematics alone. However, any differences from the results presented here are not expected to be large since the "common sense" world views held by students pre-instruction are assumed to be fairly uniform. The same can be said for classes using differing teaching methods, as this sample is a mixture of teaching methods as well.

Another limitation of this study is the unique manner in which the correlation matrix had to be handled. The treatment used in this study does cause significant changes to the factor structure found through factor analysis for the original correlation matrix. However, without this treatment, coherent world views would not be as readily identifiable since the responses within the same questions would be highly anticorrelated and would result in the factors grouping options from the same questions together. The treatment used in this study is admittedly ad hoc, but does produce conceptually understandable results that agree with past qualitative and anecdotal results $[10,22,23,29-31]$.

\section{FUTURE WORKS}

Since the data used in this study included students with a mixture of mathematical sophistication and myriad teaching techniques, a study which looks into purer set of data would be interesting. For example, studies using data of different teaching methods may find differences in the misconceptions within the data, which could be used to encourage the use of one method over another.

To strengthen the results of this study, student interviews or written responses could be used to lend support for the factor interpretations given here. For instance, one could have students take the FCI and provide written responses for all of the questions, asking them to explain their reasoning. These responses could then be thematically grouped and a fuller understanding for the distractor grouping may be possible.

Last, studies of this nature could be performed on other instruments (like the Force and Motion Conceptual Evaluation [32], the Brief Electricity and Magnetism Assessment [33], or the Conceptual Survey of Electricity and Magnetism [34]) to what kinds of alternative world views are probed within the assessments.

\section{CONCLUSIONS}

Through the utilization of an exploratory factor analysis on the response options themselves, this study sought to answer the following research questions: (i) What misconceptions are the most prevalent and coherent from a factor analysis perspective for pre- and postinstruction student responses? (ii) Which misconceptions remained unchanged, corrected, and/or were taught over the course of instruction?

The alternative world views, probed by the force concept inventory (FCI), held by a 19727 student sample were extracted from their matched pretest and post-test responses using factor analysis. The factors found on the pre- and post-test were similar in content to one another, meaning the students are likely suffering from the same misconceptions before and after instruction. The pretest factors appeared to be built more coherently around the distractor taxonomy given by the creators for the FCI, see Table XI, and the factors of the post-test seemed to be more coherently built around specific Newtonian ideas. The post-test distractor groupings were less coherent under the FCI distractor taxonomy, and were harder to describe under only one misconception. This could indicate that before instruction the students had coherent alternative world views, but these diminished over the course of instruction. As a result, many students possessed aspects of a Newtonian world view and less coherent alternative world views. The presence of the alternative world views both pre- and postinstruction is encouraging as it implies that they are not taught, but are learned by the students prior to their instruction experience.

The alternative world views found pre- and postinstruction probed misconceptions like active agent, active force, impetus as a property of objects, and the net force acting on an object determines its motion at every instant in time. 
As seen in the item response difference curves (IRDCs), many of the questions on the FCI show an overall loss of the misconception world views by the students and a growth of Newtonian conceptions. This can be seen in the IRDCs where the most commonly selected distractors in each question were selected less often post-test and the question's corresponding correct option was commonly switched into. Question 21 is an obvious exception to this trend, where students of pre-test scores below 50\% tended to switch into either a distractor or the correct answer. The quality of this question has been commented on in the past and the authors of this study suspect the question itself is not functioning properly [28]. Evidence to support the possibility that this question is malfunctioning comes from considering other items that probed the same misconception, but did not appear to behave in remotely the same manner as question 21. However, without student interview data for question 21, the claim that it is malfunctioning cannot be answered and is suggested for a future study that could produce an improved FCI as a result.

Of the alternative world views found, the IRDCs showed that the active force misconception (the idea that motion requires a cause in the form of a force) was the most 'rigid' misconception for these students.
In particular, the active force misconception shows response rate growth from pre- to post-test on five questions of the FCI. This indicates that this misconception is not consistently being fixed within the students, and is actually being promoted somehow through the instruction process. It may be that the students are leaving one novice world view for another that is more expertlike but still non-Newtonian. With these results, this study finds itself in good agreement with the results of Refs. [10,11], and suggests that instructors spend time to specifically help the students "unlearn" the alternative world views found in this study, with an emphasis placed on the active force, impetus as a property of an object, and the motion of an object being dictated by the last force, or net force, to act on the object misconceptions.

\section{ACKNOWLEDGMENTS}

The authors would like to thank Physport for supplying the data for this study, and to Jake Lindquist and Dr. Keith Johnson for reading through the manuscript and helping to improve the readability and format of the manuscript. This project was funded by the Montana State University Physics Department.

\section{APPENDIX: FCI DISTRACTOR TAXONOMY}

The appendix contains the distractor taxonomy for the FCI used in this study, see Table XI, and plots of the item response curves and item response difference curves for each of the questions on the FCI. Please note, the plots in the righthand column are on a different scale that the other two columns. This zoomed-in scale meant to enhance the readability of the middle column s plots to help identify the alternative world views that are being chosen less often postinstruction than they were preinstruciton.

TABLE XI. The following are the misconceptions paired to response options for the FCI. Questions or options that did not exist on the 1992 version, or were not included in the original taxonomy, are indicated with an asterisks as $\square^{*}$. The meaning for each of the abbreviated codings can be found below.

\begin{tabular}{|c|c|c|c|c|c|c|c|c|c|c|c|c|c|c|c|c|c|}
\hline Option & $\mathrm{Q} 1$ & Q2 & Q3 & $\mathrm{Q} 4$ & \multicolumn{2}{|c|}{ Q5* } & \multicolumn{2}{|c|}{ Q6 } & Q7 & Q8 & Q9 & $\mathrm{Q} 10$ & Q11 & Q12 & Q13 & Q14 & Q15 \\
\hline A & G3 & $\mathrm{C}$ & AF6 & AR1 & \multicolumn{2}{|c|}{$\mathrm{AF} 1^{*}$} & \multicolumn{2}{|c|}{ I5 } & I5 & CI3 & M & $\mathrm{C}$ & $\mathrm{Ob}^{*}$ & $\mathrm{CI} 2$ & I3 & R1 & $\mathrm{C}$ \\
\hline B & $\mathrm{M}$ & G3 & G4/AF5 & $\mathrm{M}$ & \multicolumn{2}{|c|}{ C } & \multicolumn{2}{|c|}{$\mathrm{C}$} & $\mathrm{C}$ & $\mathrm{C}$ & $\mathrm{CI} 3$ & I4 & $\mathrm{I} 1 / \mathrm{Ob}$ & $\mathrm{C}$ & I3 & $\mathrm{R} 1$ & AR1 \\
\hline $\mathrm{C}$ & $\mathrm{C}$ & M & $\mathrm{C}$ & $\mathrm{Ob}$ & \multicolumn{2}{|c|}{$\mathrm{AF} 2^{*}$} & \multicolumn{2}{|c|}{$\mathrm{CF}$} & $\mathrm{CI} 2 / \mathrm{CF}$ & I2 & K3 & I3 & I1 & I3 & I3 & CI2 & AR2 \\
\hline D & G3* & G3 & $\mathrm{G} 2$ & AR1 & \multicolumn{2}{|c|}{$\mathrm{AF} 2^{*}$} & \multicolumn{2}{|c|}{$\mathrm{CI} 2 / \mathrm{CF}$} & $\mathrm{I} 2 / \mathrm{I} 5 / \mathrm{CF}$ & I4 & M & I4 & $\mathrm{C}$ & $\mathrm{I} 3$ & $\mathrm{C}$ & $\mathrm{C}$ & $\mathrm{AF} 1$ \\
\hline $\mathrm{E}$ & $\mathrm{M}$ & M & G1 & $\mathrm{C}$ & \multicolumn{2}{|c|}{$\mathrm{AF} 2 / \mathrm{CF}^{*}$} & \multicolumn{2}{|c|}{ CF } & $\mathrm{CF}$ & $\mathrm{I} 2$ & $\mathrm{C}$ & M & $\mathrm{AF}^{*}$ & G5/I3 & G2 & I3 & $\mathrm{Ob}$ \\
\hline Option & Q16 & Q17 & Q1 & & Q19 & Q20 & Q21 & Q22 & Q23 & Q24 & Q25* & Q26* & Q27 & & Q28 & Q29 & Q30 \\
\hline A & $\mathrm{C}$ & CI1 & $\mathrm{AF}$ & & K2 & M & I2 & $\mathrm{AF} 4$ & I2 & $\mathrm{C}$ & M & AF4 & $\mathrm{AF} 2 / \mathrm{R} 1$ & & M & $\mathrm{Ob}$ & AF1 \\
\hline B & AR1 & $\mathrm{C}$ & c & & K1 & K2 & CI3 & $\mathrm{C}$ & $\mathrm{C}$ & M & R2 & $\mathrm{AF} 4^{*}$ & I2/R1 & & $\mathrm{AF} 1$ & $\mathrm{C}$ & I1 \\
\hline C & AR2 & $\mathrm{M}$ & $\mathrm{AF}$ & & K1 & K2 & CI2 & AF7 & CI3 & I3 & $\mathrm{C}$ & $\mathrm{I} 4^{*}$ & C & & M & $\mathrm{AF} 1$ & C \\
\hline D & $\mathrm{AF} 1$ & G1/CI1 & $\mathrm{AF}$ & & K1 & $\mathrm{C}$ & $\mathrm{I} 4$ & AF6 & $\mathrm{I} 2$ & I3* & R2 & I3* & I1 & AR & 1/AR2 & G1 & I1 \\
\hline E & $\mathrm{Ob}$ & AF1 & $\mathrm{AF} 2$ & $\mathrm{CF}^{*}$ & $\mathrm{C}$ & M & $\mathrm{C}$ & AF7 & I 2 & I3 & $\mathrm{R} 2 *$ & $\mathrm{C}$ & I4 & & $\mathrm{C}$ & AF3 & I1 \\
\hline Index & & & & & & & & & & & & Meanin & & & & & \\
\hline $\begin{array}{l}\mathrm{C} \\
\mathrm{K} 1\end{array}$ & & & & & & & & & & & & $\begin{array}{l}\text { Newton } \\
\text { position }\end{array}$ & $\begin{array}{l}\text { ian respon } \\
\text { l-velocity }\end{array}$ & $\begin{array}{l}\text { nse } \\
\text { undis }\end{array}$ & iminatec & & \\
\hline
\end{tabular}

(Table continued) 
TABLE XI. (Continued)

\begin{tabular}{|c|c|}
\hline Index & Meaning \\
\hline $\mathrm{K} 2$ & velocity-acceleration undiscriminated \\
\hline K3 & nonvectorial velocity composition \\
\hline I1 & Impetus supplied by "hit" \\
\hline $\mathrm{I} 2$ & loss or recovery of original Impetus \\
\hline I3 & Impetus dissipation \\
\hline I4 & gradual or delayed Impetus build up \\
\hline I5 & circular Impetus \\
\hline $\mathrm{AF} 1$ & only active agents exert forces \\
\hline $\mathrm{AF} 2$ & motion implies active force \\
\hline AF3 & no motion implies no force \\
\hline AF4 & velocity proportional to applied force \\
\hline AF5 & acceleration implies increasing force \\
\hline AF6 & force causes acceleration to terminal velocity \\
\hline AF7 & active force wears out \\
\hline AR1 & greater mass implies greater force \\
\hline AR2 & most active agent produces greatest force \\
\hline CI1 & largest force determines motion \\
\hline $\mathrm{CI} 2$ & force compromise determines motion \\
\hline $\mathrm{CI} 3$ & last force to act determines motion \\
\hline $\mathrm{CF}$ & centrifugal force \\
\hline $\mathrm{Ob}$ & obstacles exert no force \\
\hline $\mathrm{R} 1$ & mass makes things stop \\
\hline $\mathrm{R} 2$ & motion when force overcomes resistance \\
\hline G1 & air pressure-assisted gravity \\
\hline $\mathrm{G} 2$ & gravity intrinsic to mass \\
\hline G3 & heavier objects fall faster \\
\hline G4 & gravity increases as objects fall \\
\hline G5 & gravity acts after Impetus wears down \\
\hline M & miscellaneous options \\
\hline
\end{tabular}



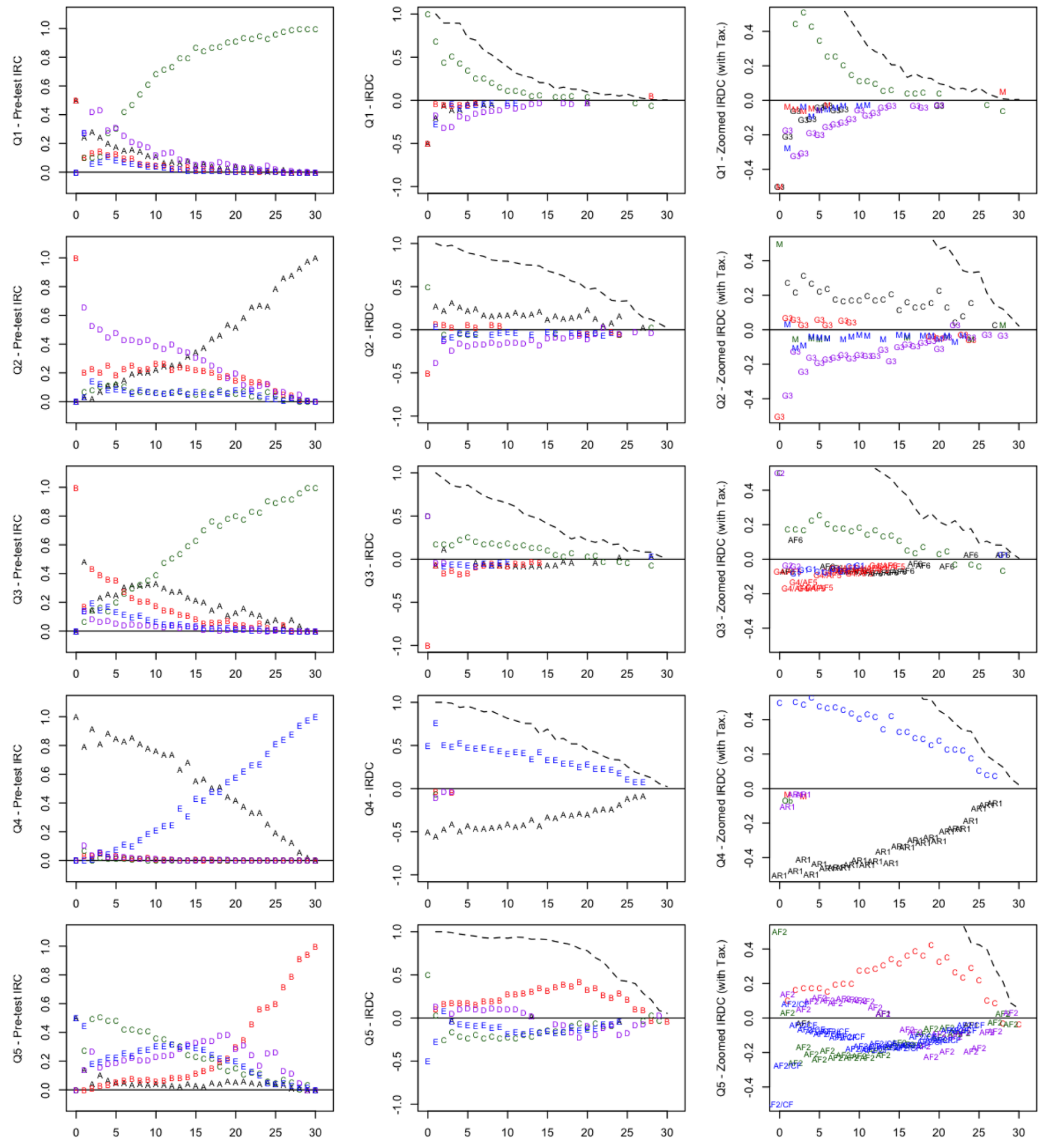

Pre-test Total Scores

FIG. 12. From left to right along one row are the pretest's item response curve, the item response difference curve, and an enlarged item response difference curve plotted using the distractors' taxonomy from Table XI. This figure, from top to bottom, displays questions $1-5$ for the FCI. 

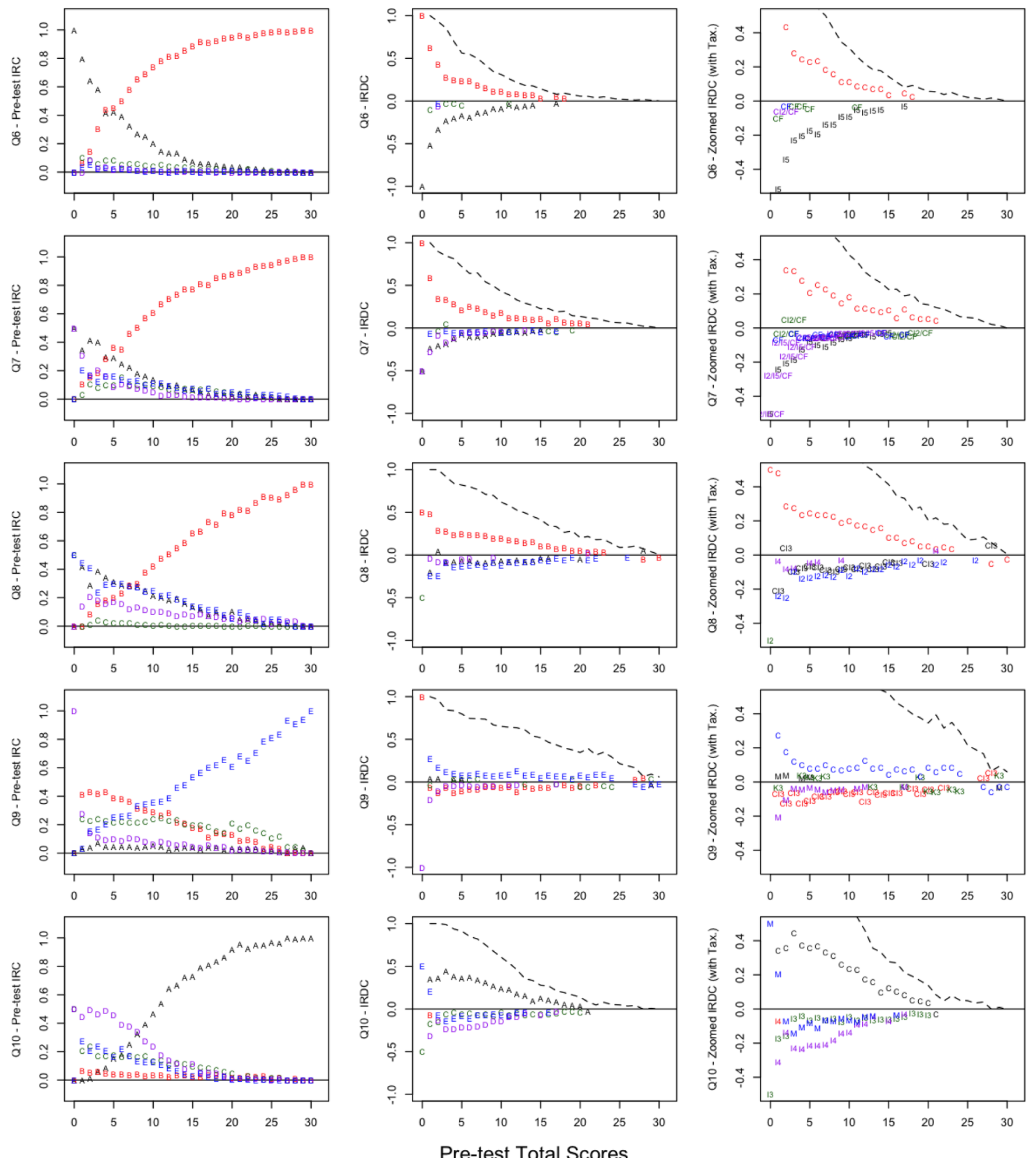

FIG. 13. From left to right along one row are the pretest's item response curve, the item response difference curve, and an enlarged item response difference curve plotted using the distractors' taxonomy from Table XI. This figure, from top to bottom, displays questions $6-10$ for the FCI. 

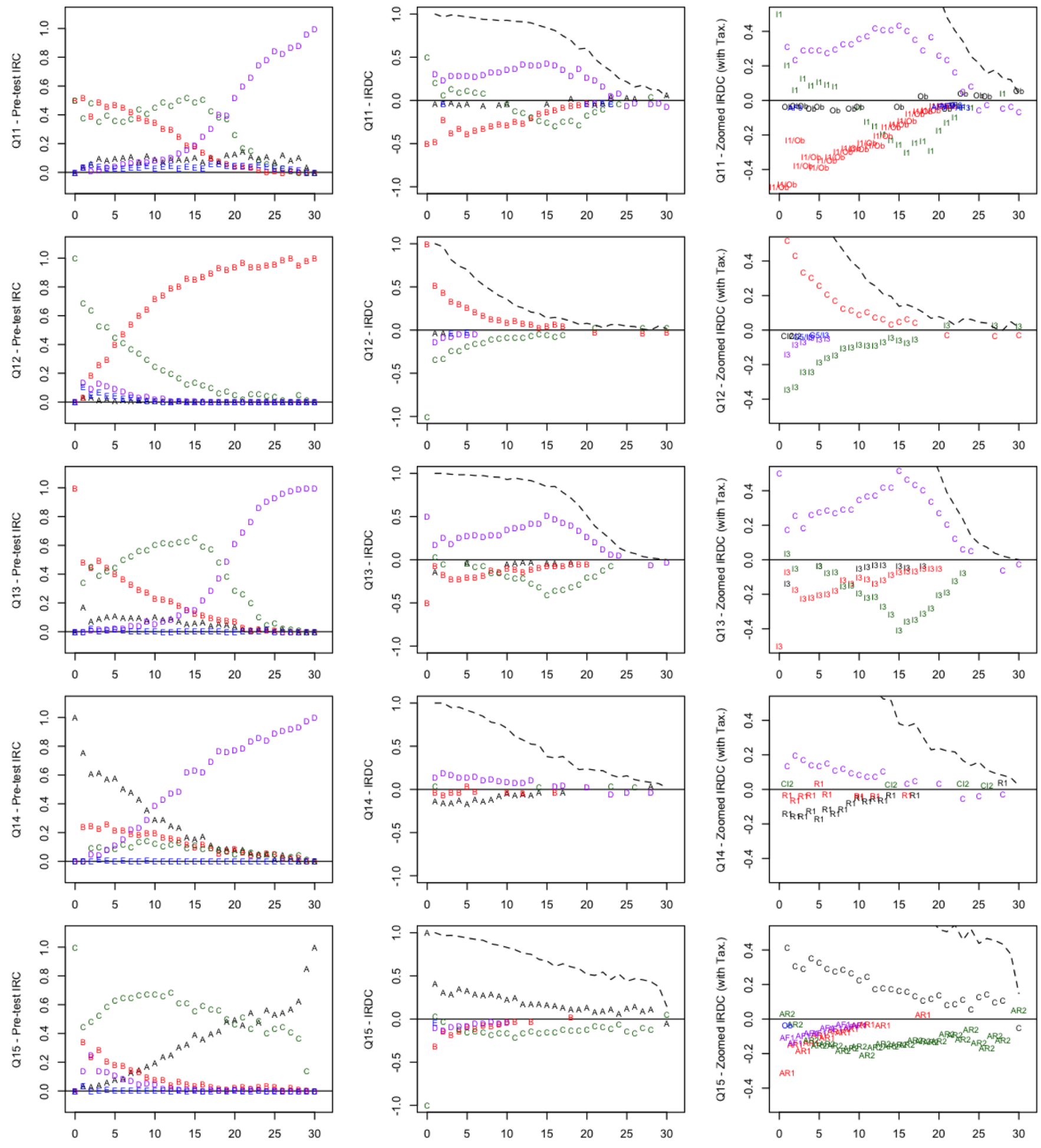

Pre-test Total Scores

FIG. 14. From left to right along one row are the pretest's item response curve, the item response difference curve, and an enlarged item response difference curve plotted using the distractors' taxonomy from Table XI. This figure, from top to bottom, displays questions $11-15$ for the FCI. 

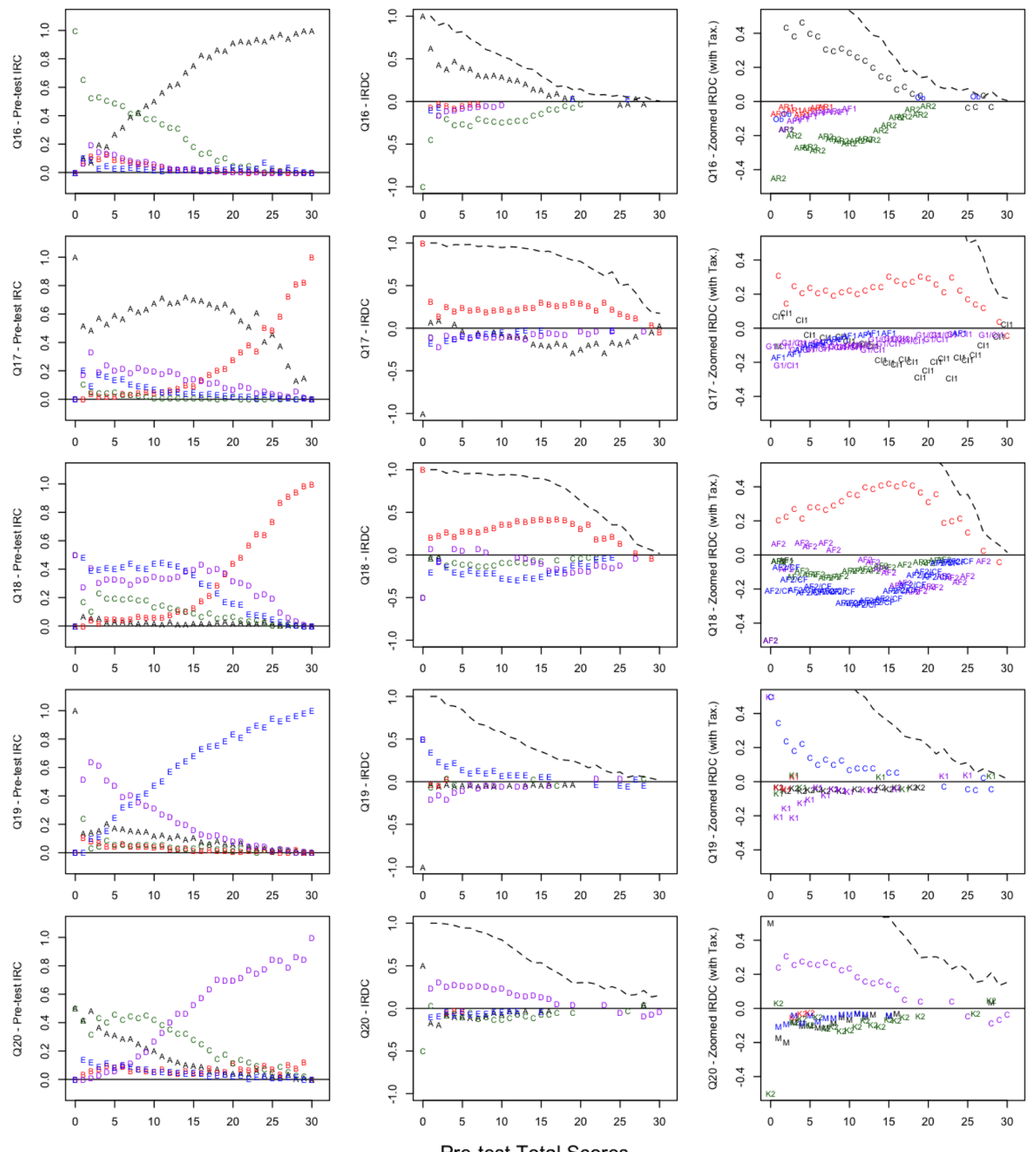

Pre-test Total Scores

FIG. 15. From left to right along one row are the pretest's item response curve, the item response difference curve, and an enlarged item response difference curve plotted using the distractors' taxonomy from Table XI. This figure, from top to bottom, displays questions 16-20 for the FCI. 

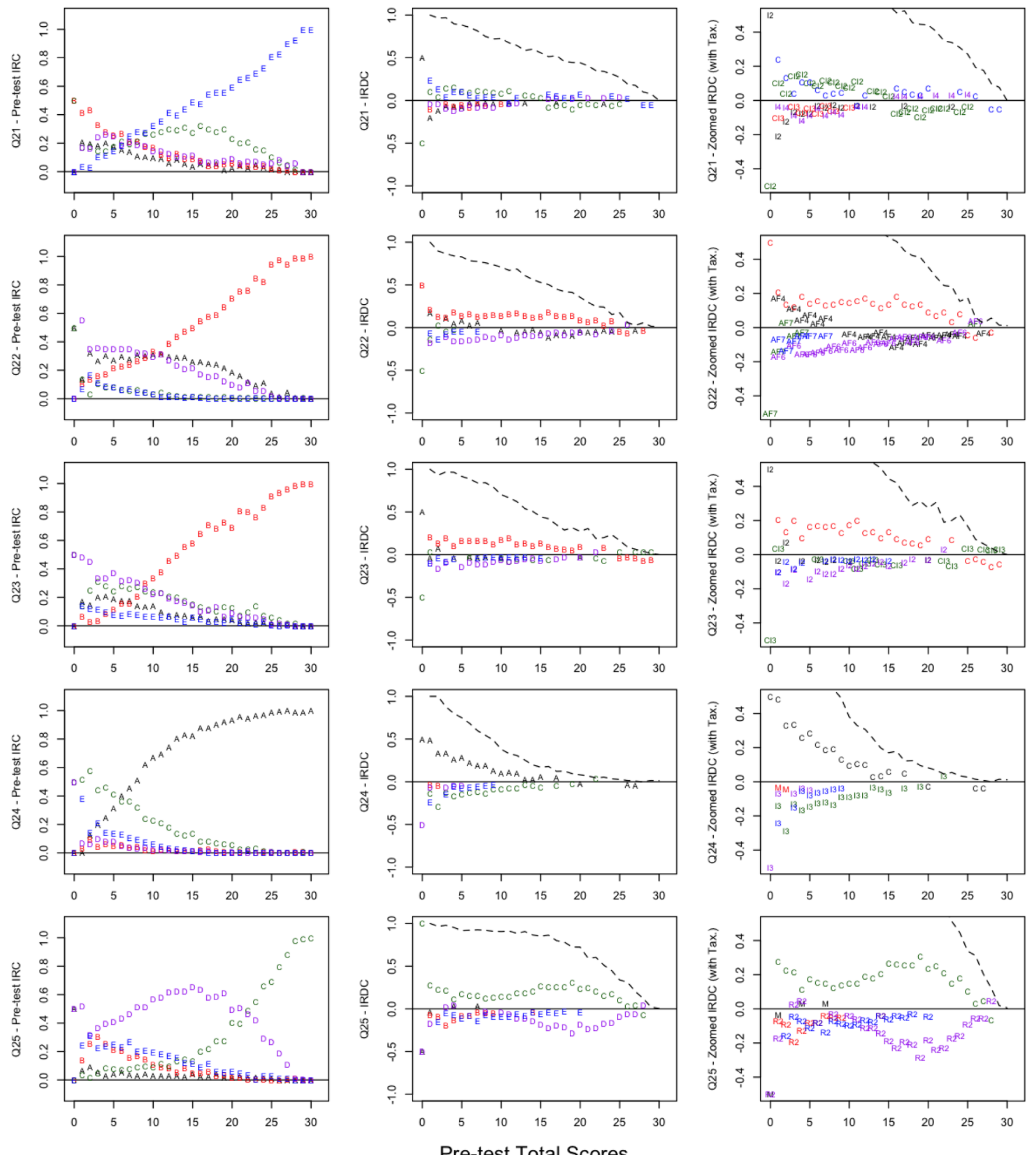

FIG. 16. From left to right along one row are the pretest's item response curve, the item response difference curve, and an enlarged item response difference curve plotted using the distractors' taxonomy from Table XI. This figure, from top to bottom, displays questions $21-25$ for the FCI. 

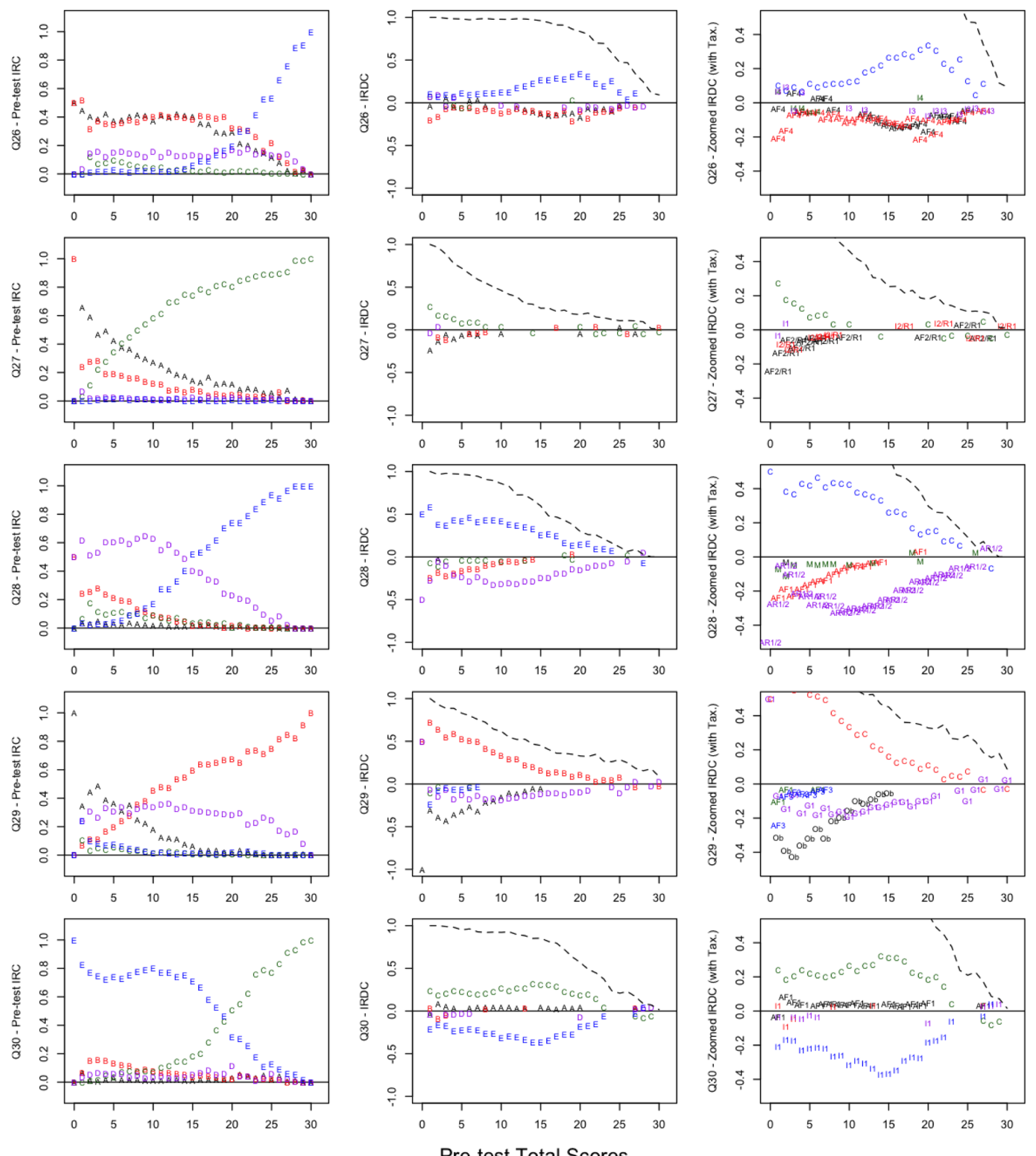

Pre-test Total Scores

FIG. 17. From left to right along one row are the pretest's item response curve, the item response difference curve, and an enlarged item response difference curve plotted using the distractors' taxonomy from Table XI. This figure, from top to bottom, displays questions $26-30$ for the FCI. 
[1] D. Hestenes, M. Wells, and G. Swackhamer, Force Concept Inventory, Phys. Teach. 30, 141 (1992).

[2] R. R. Hake, Interactive-engagement versus traditional methods: A six-thousand-student survey of mechanics test data for introductory physics courses., Am. J. Phys. 66, 64 (1998).

[3] D. Huffman and P. Heller, What does the force concept inventory actually measure?, Phys. Teach. 33, 138 (1995).

[4] M. Planinic, L. Ivanjek, and A. Susac, Rasch model based analysis of the force concept inventory., Phys. Rev. ST Phys. Educ. Res. 6, 010103 (2010).

[5] J. Wang and L. Bao, Analyzing force concept inventory with item response theory, Am. J. Phys. 78, 1064 (2010).

[6] G. A. Morris, N. Harshman, L. Branum-Martin, E. Mazur, T. Mzoughi, and S.D. Baker, An item response curves analysis of the Force Concept Inventory, Am. J. Phys. 80, 825 (2012).

[7] T. F. Scott, D. Schumayer, and A. R. Gray, Exploratory factor analysis of a Force Concept Inventory data set, Phys. Rev. ST Phys. Educ. Res. 8, 020105 (2012).

[8] T. F. Scott and D. Schumayer, Students' proficiency scores within multitrait item response theory, Phys. Rev. ST Phys. Educ. Res. 11, 020134 (2015).

[9] P. Eaton and S. D. Willoughby, Confirmatory factor analysis applied to the Force Concept Inventory, Phys. Rev. Phys. Educ. Res. 14, 010124 (2018).

[10] T. F. Scott and D. Schumayer, Conceptual coherence of non-Newtonian world views in Force Concept Inventory data, Phys. Rev. Phys. Educ. Res. 13, 010126 (2017).

[11] E. Brewe, J. Bruun, and I. G. Bearden, Using module analysis for multiple choice responses: A new method applied to force concept inventory data, Phys. Rev. Phys. Educ. Res. 12, 020131 (2016).

[12] G. A. Morris, L. Branum-Martin, N. Harshman, S. D. Baker, E. Mazur, S. Dutta, T. Mzoughi, and V. McCauley, Testing the test: Item response curves and test quality, Am. J. Phys. 74, 449 (2006).

[13] T. F. Scott and D. Schumayer, Central distractors in force concept inventory data, Phys. Rev. Phys. Educ. Res. 14, 010106 (2018).

[14] PhysPort, Security FAQ for the Assessment Data Explorer, 2017 (accessed November 20, 2017), https://www .physport.org/DataExplorer/SecurityFAQ.cfm\#2q3.

[15] P. M. Bentler and K.-H. Yuan, Positive definiteness via offdiagonal scaling of a symmetric indefinite matrix, Psychometrika 76, 119 (2011).

[16] R. Debelak and U.S. Tran, Comparing the effects of different smoothing algorithms on the assessment of dimensionality of ordered categorical items with parallel analysis, PLoS One 11, e0148143 (2016).

[17] N. J. Higham, Computing the nearest correlation matrix? A problem from finance, IMA J. Numer. Anal. 22, 329 (2002).

[18] W. Revelle, psych: Procedures for Psychological, Psychometric, and Personality Research, Northwestern University,
Evanston, Illinois, 2018, https://CRAN.R-project.org/ package $=$ psych. $\mathrm{R}$ package version 1.8.4.

[19] R. B. Cattell, The scree test for the number of factors, Multivariate Behav. Res. 1, 245 (1966).

[20] M. Ishimoto, G. Davenport, and M. C. Wittmann, Use of item response curves of the force and motion conceptual evaluation to compare Japanese and American students? Views on force and motion, Phys. Rev. Phys. Educ. Res. 13, 020135 (2017).

[21] T. Smith, K. Gray, K. Louis, B. Ricci, and N. Wright, Showing the dynamics of student thinking as measured by the FMCE, in Proceedings of the 2017 Physics Education Research Conference, Cincinnati, OH (AIP, New York, 2018), pp. 380-383.

[22] I. A. Halloun and D. Hestenes, Common sense concepts about motion, Am. J. Phys. 53, 1056 (1985).

[23] I. A. Halloun and D. Hestenes, The initial knowledge state of college physics students, Am. J. Phys. 53, 1043 (1985).

[24] J. Yasuda and M. Taniguchi, Validating two questions in the force concept inventory with subquestions, Phys. Rev. ST Phys. Educ. Res. 9, 010113 (2013).

[25] K. F. Wilson and D. J. Low, "On second thoughts...": Changes of mind as an indication of competing knowledge structures, Am. J. Phys. 83, 802 (2015).

[26] D. E. Trowbridge and L. C. McDermott, Investigation of student understanding of the concept of velocity in one dimension, Am. J. Phys. 48, 1020 (1980).

[27] D. E. Trowbridge and L. C. McDermott, Investigation of student understanding of the concept of acceleration in one dimension, Am. J. Phys. 49, 242 (1981).

[28] A. Traxler, R. Henderson, J. Stewart, G. Stewart, A. Papak, and R. Lindell, Gender fairness within the Force Concept Inventory, Phys. Rev. Phys. Educ. Res. 14, 010103 (2018).

[29] C. Terry and G. Jones, Alternative frameworks: Newton's third law and conceptual change, Eur. J. Sci. Educ. 8, 291 (1986).

[30] D. E. Brown, Students' concept of force: the importance of understanding Newton's third law, Phys. Educ. 24, 353 (1989).

[31] D. P. Maloney, Rule-governed approaches to physicsNewton's third law, Phys. Educ. 19, 37 (1984).

[32] R. K. Thornton and D. R. Sokoloff, Assessing student learning of Newton's laws: The force and motion conceptual evaluation and the evaluation of active learning laboratory and lecture curricula, Am. J. Phys. 66, 338 (1998).

[33] L. Ding, R. Chabay, B. Sherwood, and R. Beichner, Evaluating an electricity and magnetism assessment tool: Brief electricity and magnetism assessment, Phys. Rev. ST Phys. Educ. Res. 2, 010105 (2006).

[34] D. P. Maloney, T. L. O'Kuma, C. J. Hieggelke, and A. Van Heuvelen, Surveying students' conceptual knowledge of electricity and magnetism, Am. J. Phys. 69, S12 (2001). 